\title{
Frobenius groups of automorphisms and their fixed points
}

\author{
Evgeny Khukhro, Natalia Makarenko and Pavel Shumyatsky \\ Communicated by Dan Segal
}

\begin{abstract}
Suppose that a finite group $G$ admits a Frobenius group of automorphisms $F H$ with kernel $F$ and complement $H$ such that the fixed-point subgroup of $F$ is trivial: $C_{G}(F)=1$. In this situation various properties of $G$ are shown to be close to the corresponding properties of $C_{G}(H)$. By using Clifford's theorem it is proved that the order $|G|$ is bounded in terms of $|H|$ and $\left|C_{G}(H)\right|$, the rank of $G$ is bounded in terms of $|H|$ and the rank of $C_{G}(H)$, and that $G$ is nilpotent if $C_{G}(H)$ is nilpotent. Lie ring methods are used for bounding the exponent and the nilpotency class of $G$ in the case of metacyclic $F H$. The exponent of $G$ is bounded in terms of $|F H|$ and the exponent of $C_{G}(H)$ by using Lazard's Lie algebra associated with the Jennings-Zassenhaus filtration and its connection with powerful subgroups. The nilpotency class of $G$ is bounded in terms of $|H|$ and the nilpotency class of $C_{G}(H)$ by considering Lie rings with a finite cyclic grading satisfying a certain 'selective nilpotency' condition. The latter technique also yields similar results bounding the nilpotency class of Lie rings and algebras with a metacyclic Frobenius group of automorphisms, with corollaries for connected Lie groups and torsion-free locally nilpotent groups with such groups of automorphisms. Examples show that such nilpotency results are no longer true for non-metacyclic Frobenius groups of automorphisms.
\end{abstract}

Keywords. Frobenius group, automorphism, finite group, exponent, Lie ring, Lie algebra, Lie group, graded, solvable, nilpotent.

2010 Mathematics Subject Classification. Primary 17B40, 20D45; secondary 17B70, 20D15, 20E36, 20F40, 22E25.

\section{Introduction}

Suppose that a finite group $G$ admits a Frobenius group of automorphisms $F H$ with kernel $F$ and complement $H$ such that the fixed-point subgroup (which we call the centralizer) of $F$ is trivial: $C_{G}(F)=1$. Experience shows that many prop-

The second author was partly supported by the Programme of Support of Leading Scientific Schools of the Russian Federation (grant NSh-3669.2010.1). The third author was supported by CNPqBrazil. 
erties of $G$ must be close to the corresponding properties of $C_{G}(H)$. For example, when $G F$ is also a Frobenius group with kernel $G$ and complement $F$ (so that $G F H$ is a double Frobenius group), the second and third authors [19] proved that the nilpotency class of $G$ is bounded in terms of $|H|$ and the nilpotency class of $C_{G}(H)$. This result solved in the affirmative Mazurov's Problem 17.72(a) in Kourovka Notebook [25].

In this paper we derive properties of $G$ from the corresponding properties of $C_{G}(H)$ in more general settings, no longer assuming that $G F$ is also a Frobenius group. Note also that the order of $G$ is not assumed to be coprime to the order of $F H$.

By using variations on Clifford's theorem it is shown that the order $|G|$ is bounded in terms of $|H|$ and $\left|C_{G}(H)\right|$, the rank of $G$ is bounded in terms of $|H|$ and the rank of $C_{G}(H)$, and that $G$ is nilpotent if $C_{G}(H)$ is nilpotent. By using various Lie ring methods bounds for the exponent and the nilpotency class of $G$ are obtained in the case of metacyclic $F H$. For bounding the exponent, we use Lazard's Lie algebra associated with the Jennings-Zassenhaus filtration and its connection with powerful subgroups. For bounding the nilpotency class we consider Lie rings with a finite cyclic grading satisfying a certain condition of 'selective nilpotency'. The latter technique yields similar results giving nilpotency of bounded nilpotency class (also known as nilpotency index) of Lie rings and algebras with a metacyclic Frobenius group of automorphisms, with corollaries for connected Lie groups and torsion-free locally nilpotent groups with such groups of automorphisms. Examples show that such nilpotency results are no longer true for non-metacyclic Frobenius groups of automorphisms.

We now describe the results and the structure of the paper in more detail. Recall that we consider a finite group $G$ admitting a Frobenius group of automorphisms $F H$ with kernel $F$ and complement $H$ such that $C_{G}(F)=1$. It is worth noting from the outset that since $F$ is nilpotent being a Frobenius kernel, the condition $C_{G}(F)=1$ implies the solvability of $G$ by a theorem of Belyaev and Hartley [1] based on the classification of finite simple groups.

In Section 2 we begin establishing the connection between the properties of $G$ and $C_{G}(H)$ by proving that the orders satisfy the equation $|G|=\left|C_{G}(H)\right|^{|H|}$, the rank of $G$ is bounded in terms of $|H|$ and the rank of $C_{G}(H)$, and that $G$ is nilpotent if $C_{G}(H)$ is nilpotent (Theorem 2.7). These results are proved by using Clifford's theorem on the basis of information about the fixed points of $H$ in $F H$-invariant sections of $G$. In particular, we prove that these fixed points are the images of elements of $C_{G}(H)$ (Theorem 2.3), which is a non-trivial fact since the orders of $G$ and $H$ are not assumed to be coprime.

In Section 3 we deal with bounding the exponent. First we develop the requisite Lie ring technique, some of which was used earlier by the first and third authors 
in [11]. We define Lazard's Lie algebra $L_{p}(P)$ associated with the Jennings-Zassenhaus filtration of a finite $p$-group $P$ and recall Lazard's observation that elements of $P$ of order $p^{k}$ give rise to elements of $L_{p}(P)$ that are ad-nilpotent of index $p^{k}$. A useful property is the existence of a powerful subgroup of $P$ of index bounded in terms of the number of generators of $P$ and the nilpotency class of $L_{p}(P)$. A key lemma gives a bound for the nilpotency class of a finitely generated solvable Lie algebra 'saturated' with ad-nilpotent elements. The main result of the section is Theorem 3.4: if a finite group $G$ admits a Frobenius group of automorphisms $F H$ with cyclic kernel $F$ and complement $H$ such that $C_{G}(F)=1$, then the exponent of $G$ is bounded in terms of $|F H|$ and the exponent of $C_{G}(H)$. At present it is unclear, even in the case where $G F H$ is a double Frobenius group, if the bound can be made independent of $|F|$, which would give an affirmative answer to part (b) of Mazurov's Problem 17.72 in [25]. In the proof of Theorem 3.4 a reduction to finite $p$-groups is given by Dade's theorem [3]. Then nilpotency of Lazard's Lie algebra gives a reduction to powerful $p$-groups, to which a lemma from Section 2 about fixed points of $H$ is applied.

In Section 4 a different Lie ring theory is developed, which is used later in Section 5 for bounding the nilpotency class of groups and Lie rings with a metacyclic Frobenius group $F H$ of automorphisms. This theory is stated in terms of a Lie ring $L$ with a finite cyclic grading (which naturally arises from the 'eigenspaces' for $F$ ). The condition of the fixed-point subring $C_{L}(H)$ being nilpotent of class $c$ implies certain restrictions on the commutation of the grading components, which we nickname 'selective nilpotency'. For example, in [9] it was shown that if we have $C_{L}(F)=0, c=1$, and $|F|$ is a prime, then each component commutes with all but at most $(c,|H|)$-boundedly many components, which in turn implies a strong bound for the nilpotency class of $L$. For greater values of $c$ more complicated 'selective nilpotency' conditions naturally arise; similar conditions were exploited earlier in the paper [19] on double Frobenius groups.

In Section 5 we obtain bounds for the nilpotency class of groups and Lie rings with metacyclic Frobenius groups of automorphisms. (Examples show that such results are no longer true for non-metacyclic Frobenius groups of automorphism.) The main result for finite groups is Theorem 5.8: if a finite group $G$ admits a Frobenius group of automorphisms $F H$ with cyclic kernel $F$ and complement $H$ such that $C_{G}(F)=1$ and $C_{G}(H)$ is nilpotent of class $c$, then $G$ is nilpotent of class bounded in terms of $c$ and $|H|$ only. The proof is based on the analogous result for Lie rings (Theorem 5.6).

We state separately the result for Lie algebras as Theorem 5.1: if a Lie algebra $L$ over any field admits a Frobenius group of automorphisms $F H$ with cyclic kernel $F$ such that $C_{L}(F)=0$ and $C_{L}(H)$ is nilpotent of class $c$, then $L$ is nilpotent of class bounded in terms of $c$ and $|H|$ only. (Here $C_{L}(F)$ and $C_{L}(H)$ denote the 
fixed-point subalgebras for $F$ and $H$.) One corollary of this theorem is for connected Lie groups with metacyclic Frobenius groups of automorphisms satisfying similar conditions (Theorem 5.4). Another application is for torsion-free locally nilpotent groups with such groups of automorphisms (Theorem 5.5).

The induced group of automorphisms of an invariant section is often denoted by the same letter (which is a slight abuse of notation as the action may become non-faithful). We use the abbreviation, say, " $(m, n)$-bounded" for "bounded above in terms of $m, n$ only".

\section{Fixed points of Frobenius complements}

We begin with a theorem of Belyaev and Hartley [1] based on the classification of finite simple groups (see also [14]).

Theorem 2.1 ([1, Theorem 0.11]). Suppose that a finite group $G$ admits a nilpotent group of automorphisms $F$ such that $C_{G}(F)=1$. Then $G$ is solvable.

We now discuss the question of covering the fixed points of a group of automorphisms in an invariant quotient by the fixed points in the group. Let $A \leqslant$ Aut $G$ for a finite group $G$ and let $N$ be a normal $A$-invariant subgroup of $G$. It is well known that if $(|A|,|N|)=1$, then $C_{G / N}(A)=C_{G}(A) N / N$. If we do not assume that $(|A|,|N|)=1$, the equality $C_{G / N}(A)=C_{G}(A) N / N$ may no longer be true. However there are some important cases when it does hold. In particular, we have the following lemma.

Lemma 2.2. Let $G$ be a finite group admitting a nilpotent group of automorphisms $F$ such that $C_{G}(F)=1$. If $N$ is a normal $F$-invariant subgroup of $G$, then

$$
C_{G / N}(F)=1 .
$$

Proof. Since $F$ is a Carter subgroup of $G F$, it follows that $N F / N$ is a Carter subgroup of $G F / N$. Hence $C_{G / N}(F)=1$.

The following theorem was proved in [10] under the additional coprimeness assumption $(|N|,|F|)=1$, so here we only have to provide a reduction to this case.

Theorem 2.3. Suppose that a finite group $G$ admits a Frobenius group of automorphisms $F H$ with kernel $F$ and complement $H$. If $N$ is an $F H$-invariant normal subgroup of $G$ such that $C_{N}(F)=1$, then

$$
C_{G / N}(H)=C_{G}(H) N / N .
$$


Proof. As a Frobenius kernel, $F$ is nilpotent. Hence $N$ is solvable by Theorem 2.1. Consider an unrefinable $F H$-invariant normal series of $G$

$$
G>N=N_{1}>N_{2}>\cdots>N_{k}>N_{k+1}=1
$$

connecting $N$ with 1 ; its factors $N_{i} / N_{i+1}$ are elementary abelian. We apply induction on $k$ to find an element of $C_{G}(H)$ in any $g N \in C_{G / N}(H)$.

For $k>1$ consider the quotient $G / N_{k}$ and the induced group of automorphisms $F H$. By Lemma 2.2,

$$
C_{G / N_{k}}(F)=1 .
$$

By induction there is $c_{1} N_{k} \in C_{G / N_{k}}(H) \cap g N / N_{k}$, and it remains to find a required element $c \in C_{G}(H) \cap c_{1} N_{k} \subseteq C_{G}(H) \cap g N$. Thus the proof of the induction step will follow from the case $k=1$.

Let $k=1$; then $N=N_{k}$ is a $p$-group for some prime $p$. Let $F=F_{p} \times F_{p^{\prime}}$, where $F_{p}$ is the Sylow $p$-subgroup of $F$. Since $C_{N}\left(F_{p^{\prime}}\right)$ is $F_{p}$-invariant, we must actually have $C_{N}\left(F_{p^{\prime}}\right)=1$. Indeed, otherwise the $p$-group $F_{p}$ would have nontrivial fixed points on the $p$-group $C_{N}\left(F_{p^{\prime}}\right)$, and clearly $C_{C_{N}\left(F_{p^{\prime}}\right)}\left(F_{p}\right)=C_{N}(F)$ (this argument works even if $F_{p}=1$ ). Thus, the hypotheses of the theorem also hold for $G$ with the Frobenius group of automorphisms $F_{p^{\prime}} H$ satisfying the additional condition $\left(|N|,\left|F_{p^{\prime}}\right|\right)=1$. Now [10, Theorem 1] can be applied to produce a required fixed point.

We now prove a few useful lemmas about a finite group with a Frobenius group of automorphisms.

Lemma 2.4. Suppose that a finite group $G$ admits a Frobenius group of automorphisms $F H$ with kernel $F$ and complement $H$ such that $C_{G}(F)=1$. Then we have $G=\left\langle C_{G}(H)^{f} \mid f \in F\right\rangle$.

Proof. The group $G$ is solvable by Theorem 2.1. Consider an unrefinable $F H$-invariant normal series

$$
G=G_{1}>G_{2}>\cdots>G_{k}>G_{k+1}=1 .
$$

It is clearly sufficient to prove that every factor $S=G_{i} / G_{i+1}$ of this series is covered by $\left\langle C_{G_{i}}(H)^{f} \mid f \in F\right\rangle$, that is,

$$
\left\langle C_{G_{i}}(H)^{f} \mid f \in F\right\rangle G_{i+1} / G_{i+1}=G_{i} / G_{i+1} .
$$

By Theorem 2.3, this is the same as $\left\langle C_{S}(H)^{f} \mid f \in F\right\rangle=S$. Recall that we have $C_{S}(F)=1$ by Lemma 2.2. Then Clifford's theorem can be applied to show that $C_{S}(H) \neq 1$. 
Recall that for a group $A$ and a field $k$, a free $k A$-module of dimension $n$ is a direct sum of $n$ copies of the group algebra $k A$, each of which can be regarded as a vector space over $k$ of dimension $|A|$ with a basis $\left\{v_{g} \mid g \in A\right\}$ labelled by elements of $A$ on which $A$ acts in a regular representation: $v_{g} h=v_{g h}$. By the Deuring-Noether theorem [2, Theorem 29.7] two representations over a smaller field are equivalent if they are equivalent over a larger field; therefore being a free $k A$-module is equivalent to being a free $\bar{k} A$-module for any field extension $\bar{k} \supseteq k$, as the corresponding permutational matrices are defined over the prime field.

We denote by $\mathbb{F}_{p}$ the field of $p$ elements.

Lemma 2.5. Each factor $S$ of (2.1) is a free $\mathbb{F}_{p} H$-module for the appropriate prime $p$.

Proof. Again, we only provide reduction to the coprime case considered in [10]. Let $S$ be an elementary $p$-group; then let $F=F_{p} \times F_{p^{\prime}}$ as in the proof of Theorem 2.3 above. As therein, we must actually have $C_{S}\left(F_{p^{\prime}}\right)=1$. Refining $S$ by a non-refinable $F_{p^{\prime}} H$-invariant normal series we obtain factors that are irreducible $\mathbb{F}_{p} F_{p^{\prime}} H$-modules. Having the additional condition that $p \nmid\left|F_{p^{\prime}}\right|$ we can now apply, for example, [10, Lemma 2] to obtain that each of them is a free $\mathbb{F}_{p} H$-module, and therefore $S$ is also a free $\mathbb{F}_{p} H$-module.

We now finish the proof of Lemma 2.4. By Lemma 2.5, $S$ is a free $\mathbb{F}_{p} H$-module, which means that $S=\bigoplus_{h \in H} T h$ for some $\mathbb{F}_{p} H$-submodule $T$. Hence we have $C_{S}(H) \neq 0$, as $0 \neq \sum_{h \in H} t h \in C_{S}(H)$ for any $0 \neq t \in T$. Since the series (2.1) is non-refinable, the $\mathbb{F}_{p} F H$-module $S$ is irreducible. Therefore,

$$
0 \neq\left\langle C_{S}(H)^{H F}\right\rangle=\left\langle C_{S}(H)^{F}\right\rangle=S,
$$

which is exactly what we need.

Lemma 2.6. Suppose that a finite group $G$ admits a Frobenius group of automorphisms $F H$ with kernel $F$ and complement $H$ such that $C_{G}(F)=1$. Then for each prime p dividing $|G|$ there is a unique $F H$-invariant Sylow p-subgroup of $G$.

Proof. Recall that $G$ is solvable, and so is $G F$. As $F$ is a Carter subgroup of $G F$, it contains a system normalizer of $G$. By P. Hall's theorem [22, Theorem 9.2.6], a system normalizer covers all central factors of any chief series of $G F$. Since $F$ is nilpotent, it follows that $F$ is a system normalizer. Furthermore, $F$ normalizes a unique Sylow $p$-subgroup. Indeed, if $P$ and $P^{g}$ for $g \in G$ are two Sylow $p$-subgroups normalized by $F$, then $P$ is normalized by $F$ and $F^{g^{-1}}$. Then $F$ and $F^{g^{-1}}$ are Carter subgroups of $N_{G}(P)$ and $F=F^{g^{-1} n}$ for some $n \in N_{G}(P)$, whence $g^{-1} n=1$, as $N_{G}(F)=C_{G}(F)=1$. Thus, $P^{g}=P^{n}=P$. Since $F$ is normal in $F H$, the uniqueness of $P$ implies that it is also $H$-invariant. 
We now establish the connection between the order, rank, and nilpotency of $G$ and $C_{G}(H)$ for a finite group $G$ admitting a Frobenius group of automorphisms $F H$ with fixed-point-free kernel $F$. (By the rank we mean the minimum number $r$ such that every subgroup can be generated by $r$ elements.)

Theorem 2.7. Suppose that a finite group $G$ admits a Frobenius group of automorphisms $F H$ with kernel $F$ and complement $H$ such that $C_{G}(F)=1$. Then

(a) $|G|=\left|C_{G}(H)\right|^{|H|}$,

(b) the rank of $G$ is bounded in terms of $|H|$ and the rank of $C_{G}(H)$,

(c) if $C_{G}(H)$ is nilpotent, then $G$ is nilpotent.

Proof. (a) It is sufficient to prove this equality for each factor $S=G_{i} / G_{i+1}$ of the series (2.1), since

$$
\left|C_{G}(H)\right|=\prod_{i}\left|C_{G_{i} / G_{i+1}}(H)\right|
$$

by Theorem 2.3. By Lemma $2.5, S$ is a free $\mathbb{F}_{p} H$-module, which means that

$$
S=\bigoplus_{h \in H} T h
$$

for some $\mathbb{F}_{p} H$-submodule $T$. Therefore, we have $C_{S}(H)=\left\{\sum_{h \in H} t h \mid t \in T\right\}$ and $\left|C_{S}(H)\right|=|T|$, whence $|S|=|T|^{|H|}$.

(b) It is known that the rank of a finite (solvable) group $G$ is bounded in terms of the maximum rank of its Sylow subgroups ([12]). Let $P$ be an $F H$-invariant Sylow $p$-subgroup of $G$ given by Lemma 2.6. It is known that the rank of a $p$-group of automorphisms of a finite $p$-group $U$ is bounded in terms of the rank of $U$. Let $U$ denote a Thompson critical subgroup of $P$; recall that $U$ is a characteristic subgroup of nilpotency class at most 2 containing its centralizer in $P$ (see, for example, [5, Theorem 5.3.11]). Thus the rank of $P$ is bounded in terms of the rank of $U$. In turn, since $U$ is nilpotent of class at most 2, the rank of $U$ is bounded in terms of the rank of $S=U / \Phi(U)$. The group $S$ can be regarded as an $\mathbb{F}_{p} F H$-module, which is a free $\mathbb{F}_{p} H$-module by a repeated application of Lemma 2.5 to an unrefinable series of $\mathbb{F}_{p} F H$-submodules of $U / \Phi(U)$. By the same argument as in the proof of (a) above, the rank of $S$ is equal to $|H|$ times the rank of $C_{S}(H)$. By Theorem 2.3, $C_{S}(H)$ is covered by $C_{G}(H)$; as a result, the rank of $S$ is at most $|H|$ times the rank of $C_{G}(H)$.

(c) First we make a simple remark that in any action of the Frobenius group $F H$ with non-trivial action of $F$ the complement $H$ acts faithfully. Indeed, the kernel $K$ that does not contain $F$ must intersect $H$ trivially: $K \cap H$ acts trivially on 
$F /(K \cap F) \neq 1$ and therefore has non-trivial fixed points on $F$, as the action is coprime.

Suppose that $C_{G}(H)$ is nilpotent. We prove that then $G$ is nilpotent by contradiction, considering a counterexample $G F H$ of minimal possible order. Recall that $G$ is solvable. Suppose that $G$ is not nilpotent; then it is easy to find an $F H$-invariant section $V U$ of $G$ such that $V$ and $U$ are elementary abelian groups of coprime orders, $V$ is normal in $V U F H$, and $U$ acts faithfully on $V$ with $C_{V}(U)=1$. Note that $C_{V U}(F)=1$ by Lemma 2.2. In particular, $F$ acts non-trivially on $V U$ and therefore $H$ acts faithfully on $V U$. Since $C_{V U}(H)$ is covered by $C_{G}(H)$ by Theorem 2.3, it follows that $C_{V U}(H)$ is nilpotent. Thus, we can replace $G$ by $U V$, and $F$ by its image in its action on $U V$, so by the minimality of our counterexample we must actually have $G=V U$.

Also by Theorem 2.3, $C_{V U}(H)=C_{V}(H) C_{U}(H)$. Furthermore, since $U$ and $V$ have coprime orders, the nilpotency of $C_{V U}(H)$ implies that it is abelian; in other words, $C_{U}(H)$ centralizes $C_{V}(H)$. Note that $C_{U}(H) \neq 1$ by Lemma 2.4 (or 2.5).

Let $V$ be an elementary $p$-group; we can regard $V$ as an $\mathbb{F}_{p} U F H$-module. Note that $V$ is a free $\mathbb{F}_{p} H$-module by Lemma 2.5. We extend the ground field $\mathbb{F}_{p}$ to a finite field $\overline{\mathbb{F}}_{p}$ that is a splitting field for $U F H$ and obtain an $\overline{\mathbb{F}}_{p} U F H$-module

$$
\widetilde{V}=V \otimes_{\mathbb{F}_{p}} \overline{\mathbb{F}}_{p} .
$$

Many of the above-mentioned properties of $V$ are inherited by $\widetilde{V}$ :

(V1) $\widetilde{V}$ is a faithful $\overline{\mathrm{F}}_{p} U$-module,

(V2) $C_{\widetilde{V}}(U)=0$,

(V3) $C_{U}(H)$ acts trivially on $C_{\widetilde{V}}(H)$,

(V4) $C_{\widetilde{V}}(F)=0$,

(V5) $\widetilde{V}$ is a free $\overline{\mathbb{F}}_{p} H$-module.

Consider an unrefinable series of $\overline{\mathrm{F}}_{p} U F H$-submodules

$$
\widetilde{V}=V_{1}>V_{2}>\cdots>V_{k}>V_{k+1}=0 .
$$

Suppose that $W$ is one of the factors of this series; it is a non-trivial irreducible $\overline{\mathbb{F}}_{p} U F H$-submodule. Note that $C_{W}(F)=0$ by Lemma 2.2, as we can still regard $\widetilde{V}$ as a finite (additive) group on which $F$ acts fixed-point-freely by property (V4). For the same reason, $W$ is a free $\overline{\mathbb{F}}_{p} H$-module by Lemma 2.5 .

Let

$$
W=W_{1} \oplus \cdots \oplus W_{t}
$$


be the decomposition of $W$ into the direct sum of Wedderburn components $W_{i}$ with respect to $U$. On each of the $W_{i}$ the group $U$ is represented by scalar multiplications. We consider the transitive action of $F H$ on the set $\Omega=\left\{W_{1}, \ldots, W_{t}\right\}$.

Lemma 2.8. All orbits of $H$ on $\Omega$, except for possibly one, are regular (that is, of length $|H|)$.

Proof. First note that $H$ transitively permutes the $F$-orbits on $\Omega$. Let $\Omega_{1}$ be one of these $F$-orbits and let $H_{1}$ be the stabilizer of $\Omega_{1}$ in $H$. If $H_{1}=1$, then all the $H$-orbits are regular, so we assume that $H_{1} \neq 1$. We claim that $H_{1}$ has exactly one non-regular orbit on $\Omega_{1}$ (actually, a fixed point).

Let $\bar{F}$ be the image of $F$ in its action on $\Omega_{1}$. If $\bar{F}=1$, then $\Omega_{1}$ consists of a single Wedderburn component, on which $U$ acts by scalar multiplications, and acts non-trivially by property (V2). Then $F$ acts trivially on the non-trivial quotient of $U$ by the corresponding kernel, which contradicts Lemma 2.2. Thus, $\bar{F} \neq 1$, and by the remark at the beginning of the proof, $\bar{F} H_{1}$ is a Frobenius group with complement $H_{1}$.

Let $S$ be the stabilizer of a point in $\Omega_{1}$ in $\bar{F} H_{1}$. Since $\left|\Omega_{1}\right|=|\bar{F}: \bar{F} \cap S|=$ $\left|\bar{F} H_{1}: S\right|$ and the orders $|\bar{F}|$ and $\left|H_{1}\right|$ are coprime, $S$ contains a conjugate of $H_{1}$; without loss of generality we assume that $H_{1} \leqslant S$. Any other stabilizer of a point is equal to $S^{f}$ for $f \in \bar{F} \backslash S$. We claim that

$$
S^{f} \cap H_{1}=1,
$$

which is the same as $S \cap H_{1}^{f^{-1}}=1$. But all the conjugates $H_{1}^{x}$ for $x \in \bar{F}$ are distinct and disjoint and their union contains all the elements of $\bar{F} H_{1}$ of orders dividing $\left|H_{1}\right|$; the same is true for the conjugates of $H_{1}$ is $S$. Therefore the only conjugates of $H_{1}$ intersecting $S$ are $H_{1}^{s}$ for $s \in S$.

The $H$-orbits of elements of regular $H_{1}$-orbits are regular $H$-orbits. Thus there is one non-regular $H$-orbit on $\Omega$ - the $H$-orbit of the fixed point of $H_{1}$ on $\Omega_{1}$.

Consider any regular $H$-orbit on the set $\Omega$, which we temporarily denote by $\left\{W_{h} \mid h \in H\right\}$. Let $X=\bigoplus_{h \in H} W_{h}$. Then, as before,

$$
C_{X}(H)=\left\{\sum_{h \in H} x h \mid x \in W_{1}\right\} .
$$

Since $C_{U}(H)$, as a subgroup of $U$, acts on each $W_{h}$ by scalar multiplications and centralizes $C_{X}(H)$ by property (V3), we obtain that, in fact, $C_{U}(H)$ must act trivially on $X$.

The sum of the $W_{i}$ over all regular $H$-orbits is obviously a free $\overline{\mathbb{F}}_{p} H$-module. Since $\widetilde{V}$ is also a free $\overline{\mathbb{F}}_{p} H$-module by property (V5), the sum $Y$ of the $W_{i}$ over 
the only one, by Lemma 2.8 , possibly remaining non-regular $H$-orbit must also be a free $\overline{\mathbb{F}}_{p} H$-module. Let $Y=\bigoplus_{h \in H} Z h$ for some $\overline{\mathbb{F}}_{p} H$-submodule $Z$. Then, as before,

$$
C_{Y}(H)=\left\{\sum_{h \in H} y h \mid y \in Z\right\} .
$$

The subgroup $C_{U}(H)$ acts on each $W_{i}$ by scalar multiplications. Since $Y$ is the sum over an $H$-orbit and $H$ centralizes $C_{U}(H)$, all the $W_{i}$ in the $H$-orbit are isomorphic $\overline{\mathbb{F}}_{p} C_{U}(H)$-modules. Hence $C_{U}(H)$ acts by scalar multiplications on the whole $Y$. Since $C_{U}(H)$ centralizes $C_{Y}(H)$ by property (V3), it follows that, in fact, $C_{U}(H)$ must act trivially on $Y$.

As a result, $C_{U}(H)$ acts trivially on $W$. Since this is true for every factor of the series (2.2) and the order of $U$ is coprime to the characteristic $p$ (or to the order of $\widetilde{V}$ ), it follows that $C_{U}(H)$ acts trivially on $\widetilde{V}$, contrary to property (V1). This contradiction completes the proof.

\section{Bounding the exponent}

Here we bound the exponent of a group with a metacyclic Frobenius group of automorphisms. But first we develop the requisite Lie ring technique. The following general definitions are also used in subsequent sections.

The Lie subring (or subalgebra) generated by a subset $U$ is denoted by $\langle U\rangle$, and the ideal generated by $U$ by id $\langle U\rangle$. Products in a Lie ring are called commutators. We use the term "span" both for the subspace (in the case of algebras) and for the additive subgroup generated by a given subset. For subsets $X, Y$ we denote by $[X, Y]$ the span of all commutators $[x, y], x \in X, y \in Y$; this is an ideal if $X, Y$ are ideals. Terms of the derived series of a Lie ring $L$ are defined as $L^{(0)}=L$, $L^{(k+1)}=\left[L^{(k)}, L^{(k)}\right]$. Then $L$ is solvable of derived length at most $n$ (sometimes called "solvability index") if $L^{(n)}=0$. Terms of the lower central series of $L$ are defined as $\gamma_{1}(L)=L, \gamma_{k+1}(L)=\left[\gamma_{k}(L), L\right]$. Then $L$ is nilpotent of class at most $c$ (sometimes called "nilpotency index") if $\gamma_{c+1}(L)=0$. We use the standard notation for simple (left-normed) commutators:

$$
\left[x_{1}, x_{2}, \ldots, x_{k}\right]=\left[\ldots\left[\left[x_{1}, x_{2}\right], x_{3}\right], \ldots, x_{k}\right]
$$

(here the $x_{i}$ may be elements or subsets).

We use several times the following fact, which helps in proving nilpotency of a solvable Lie ring $L$ : let $K$ be an ideal of a Lie ring $L$;

$$
\text { if } \gamma_{c+1}(L) \subseteq[K, K] \text { and } \gamma_{k+1}(K)=0 \text {, then } \gamma_{c\left(\begin{array}{c}
k_{2}+1 \\
x^{2}
\end{array}\right)-\left(\begin{array}{c}
k \\
2
\end{array}\right)+1}(L)=0 \text {. }
$$


This can be regarded as a Lie ring analogue of P. Hall's theorem [6] (with a simpler 'linear' proof; see also [24] for the best possible bound for the nilpotency class of $L$ ).

Let $A$ be an additively written abelian group. A Lie ring $L$ is $A$-graded if

$$
L=\bigoplus_{a \in A} L_{a} \quad \text { and } \quad\left[L_{a}, L_{b}\right] \subseteq L_{a+b}, a, b \in A,
$$

where the $L_{a}$ are subgroups of the additive group of $L$. Elements of the grading components $L_{a}$ are called homogeneous, and commutators in homogeneous elements homogeneous commutators. An additive subgroup $H$ of $L$ is called homogeneous if $H=\bigoplus_{a}\left(H \cap L_{a}\right)$; we then write $H_{a}=H \cap L_{a}$. Clearly, any subring or ideal generated by homogeneous additive subgroups is homogeneous. A homogeneous subring and the quotient by a homogeneous ideal can be regarded as $A$-graded Lie rings with the induced gradings. Also, it is not difficult to see that if $H$ is homogeneous, then so is $C_{L}(H)$, the centralizer of $H$, which is, as usual, equal to the set $\{l \in L \mid[l, h]=0$ for all $h \in H\}$.

An element $y$ of a Lie algebra $L$ is called ad-nilpotent if there exists a positive integer $n$ such that $(\operatorname{ad} y)^{n}=0$, that is,

$$
[x, \underbrace{y, \ldots, y}_{n}]=0 \quad \text { for all } x \in L .
$$

If $n$ is the least integer with this property, then we say that $y$ is ad-nilpotent of index $n$.

Throughout the rest of this section $p$ will denote an arbitrary but fixed prime. Let $G$ be a group. We set

$$
D_{i}=D_{i}(G)=\prod_{j p^{k} \geqslant i} \gamma_{j}(G)^{p^{k}}
$$

The subgroups $D_{i}$ form the Jennings-Zassenhaus filtration

$$
G=D_{1} \geqslant D_{2} \geqslant \cdots
$$

of the group $G$. This series satisfies the inclusions

$$
\left[D_{i}, D_{j}\right] \leqslant D_{i+j} \quad \text { and } \quad D_{i}^{p} \leqslant D_{p i} \quad \text { for all } i, j .
$$

These properties make it possible to construct a Lie algebra $D L(G)$ over $\mathbb{F}_{p}$, the field with $p$ elements. Namely, consider the quotients $D_{i} / D_{i+1}$ as linear spaces over $\mathbb{F}_{p}$, and let $D L(G)$ be the direct sum of these spaces. Commutation in the 
group $G$ induces a binary operation $[\cdot, \cdot]$ in $D L(G)$. For homogeneous elements $x D_{i+1} \in D_{i} / D_{i+1}$ and $y D_{j+1} \in D_{j} / D_{j+1}$ the operation is defined by

$$
\left[x D_{i+1}, y D_{j+1}\right]=[x, y] D_{i+j+1} \in D_{i+j} / D_{i+j+1}
$$

and extended to arbitrary elements of $D L(G)$ by linearity. It is easy to check that the operation is well-defined and that $D L(G)$ with the operations + and $[\cdot, \cdot]$ is a Lie algebra over $\mathbb{F}_{p}$, which is $\mathbb{Z}$-graded with the $D_{i} / D_{i+1}$ being the grading components.

For any $x \in D_{i} \backslash D_{i+1}$ let $\bar{x}$ denote the element $x D_{i+1}$ of $D L(G)$.

Lemma 3.1 (Lazard [15]). For any $x \in G$ we have $(\operatorname{ad} \bar{x})^{p}=\operatorname{ad} \overline{x^{p}}$. Consequently, if $x$ is of finite order $p^{t}$, then $\bar{x}$ is ad-nilpotent of index at most $p^{t}$.

Let $L_{p}(G)=\left\langle D_{1} / D_{2}\right\rangle$ be the subalgebra of $D L(G)$ generated by $D_{1} / D_{2}$; it is also $\mathbb{Z}$-graded with grading components $L_{i}=L_{p}(G) \cap D_{i} / D_{i+1}$. The following lemma goes back to Lazard [16]; in the present form it can be found, for example, in [11].

Lemma 3.2. Suppose that $X$ is a $d$-generator finite $p$-group such that the Lie algebra $L_{p}(X)$ is nilpotent of class $c$. Then $X$ has a powerful characteristic subgroup of $(p, c, d)$-bounded index.

Recall that powerful $p$-groups were introduced by Lubotzky and Mann in [17]: a finite $p$-group $G$ is powerful if $G^{p} \geqslant[G, G]$ for $p \neq 2$ (or $G^{4} \geqslant[G, G]$ for $p=2$ ). These groups have many nice properties, so that often a problem becomes much easier once it is reduced to the case of powerful $p$-groups. The above lemma is quite useful as it allows us to perform such a reduction. We will also require the following lemma. Note that when a $\mathbb{Z}$-graded Lie algebra $L$ is generated by the component $L_{1}$, that is, $L=\left\langle L_{1}\right\rangle$, then in fact $L=L_{1} \oplus L_{2} \oplus \cdots$.

Lemma 3.3. Let $L=\bigoplus L_{i}$ be a $\mathbb{Z}$-graded Lie algebra over a field such that $L=\left\langle L_{1}\right\rangle$ and assume that every homogeneous component $L_{i}$ is spanned by elements that are ad-nilpotent of index at most $r$. Suppose further that $L$ is solvable of derived length $k$ and that the component $L_{1}$ has finite dimension $d$. Then $L$ is nilpotent of $(d, r, k)$-bounded class.

Proof. Without loss of generality we can assume that $k \geqslant 2$ and use induction on $k$. Let $M$ be the last non-trivial term of the derived series of $L$. By induction we assume that $L / M$ is nilpotent of $(d, r, k)$-bounded class. In particular, it follows that the dimension of $L / M$ is finite and $(d, r, k)$-bounded. If $M \leqslant Z(L)$, then we deduce easily that $L$ is nilpotent of $(d, r, k)$-bounded class. So assume that $M$ satisfies $M \nless Z(L)$. Let $j$ be the biggest index such that the component $L_{j}$ is not 
contained in $C_{L}(M)$. Thus, $K=L_{j}+C_{L}(M)$ is a non-abelian ideal in $L$. Since the dimension of $L / M$ is finite and $(d, r, k)$-bounded, there exist boundedly many elements $a_{1}, \ldots, a_{m} \in L_{j}$ such that $K=\left\langle a_{1}, \ldots, a_{m}, C_{L}(M)\right\rangle$ and each of the elements $a_{1}, \ldots, a_{m}$ is ad-nilpotent of index at most $r$. Taking into account that $j$ here is $(d, r, k)$-bounded, we can use backward induction on $j$. Therefore, by induction $L /[K, K]$ is nilpotent of $(d, r, k)$-bounded class.

Set $s=m(r-1)+1$ and consider the ideal $S=[M, K, \ldots, K]$, where $K$ occurs $s$ times. Then $S$ is spanned by commutators of the form $\left[m, b_{1}, \ldots, b_{s}\right]$ where $m \in M$ and $b_{1}, \ldots, b_{s}$ are not necessarily distinct elements from $\left\{a_{1}, \ldots, a_{m}\right\}$. Since the number $s$ is big enough, it is easy to see that there are $r$ indices $i_{1}, \ldots, i_{r}$ such that $b_{i_{1}}=\cdots=b_{i_{r}}$. Taking into account that $K / C_{L}(M)$ is abelian, we remark that $[m, x, y]=[m, y, x]$ for all $m \in M$ and $x, y \in K$. Therefore we can assume without loss of generality that $b_{1}=\cdots=b_{r}$. Since $b_{1}$ is ad-nilpotent of index at most $r$, it follows that $\left[m, b_{1}, \ldots, b_{s}\right]=0$. Thus we have $S=0$, which means that $M$ is contained in the $s$ th term of the upper central series of $K$. The fact that $L / M$ is nilpotent of $(d, r, k)$-bounded class now implies that $K$ is nilpotent of $(d, r, k)$-bounded class as well. Combining this with the fact that $L /[K, K]$ is also nilpotent of $(d, r, k)$-bounded class and using the Lie ring analogue (3.1) of P. Hall's theorem, we deduce that $L$ is also nilpotent of $(d, r, k)$-bounded class. The proof is complete.

We now prove the main result of this section.

Theorem 3.4. Suppose that a finite Frobenius group $F H$ with cyclic kernel $F$ and complement $H$ acts on a finite group $G$ in such a manner that $C_{G}(F)=1$ and $C_{G}(H)$ has exponent $e$. Then the exponent of $G$ is bounded solely in terms of $e$ and $|F H|$.

Proof. By Theorem 2.1, $G$ is solvable. The nilpotent length (Fitting height) of $G$ is bounded in terms of $|F|$ by Dade's theorem [3]. Therefore it is sufficient to bound the exponents of the factors of the Fitting series of $G$. By Lemma 2.2 and Theorem 2.3 each of them inherits the hypotheses $C_{G}(F)=1$ and $C_{G}(H)^{e}=1$. Therefore we can assume from the outset that $G$ is a finite $p$-group for some prime $p$. In view of Lemma $2.4, G=\left\langle C_{G}(H)^{f} \mid f \in F\right\rangle$, so $p$ divides $e$. We assume without loss of generality that $e$ is a $p$-power. Let $x \in G$. It is clear that $x$ is contained in an $F H$-invariant subgroup of $G$ with at most $|F H|$ generators. Therefore without loss of generality we can assume that $G$ is $|F H|$-generated.

Any group of automorphisms of the group $G$ acts naturally on every factor of the Jennings-Zassenhaus filtration of $G$. This action induces an action by automorphisms on the Lie algebra $L=L_{p}(G)$. Lemma 2.2 shows that $F$ is fixed-pointfree on every factor of the Jennings-Zassenhaus filtration. Hence $C_{L}(F)=0$. 
Kreknin's theorem [13] now tells us that $L$ is solvable of $|F|$-bounded derived length.

Every homogeneous component $L_{i}$ of the Lie algebra $L$ can be regarded as an $F H$-invariant subgroup of the corresponding quotient $D_{i} / D_{i+1}$ of the JenningsZassenhaus series. By Lemma 2.4 we obtain that $L_{i}$ is generated by the centralizers $C_{L_{i}}(H)^{f}$ where $f$ ranges through $F$. Moreover, Theorem 2.3 implies that every element of $C_{L_{i}}(H)^{f}$ is the image of some element of $C_{G}(H)^{f}$, the order of which divides $e$. It follows by Lemma 3.1 that the additive group $L_{i}$ is generated by elements that are ad-nilpotent of index at most $e$. We now deduce from Lemma 3.3 that $L$ is nilpotent of $(e,|F H|)$-bounded class.

Lemma 3.2 now tells us that the group $G$ has a powerful characteristic subgroup of $(e,|F H|)$-bounded index. It suffices to bound the exponent of this powerful subgroup so we can just assume that $G$ is powerful. Powerful $p$-groups have the property that if a powerful $p$-group $G$ is generated by elements of order dividing $p^{k}$, then the exponent of $G$ also divides $p^{k}$ (see [4, Lemma 2.2.5]). Combining this with the fact that our group $G$ is generated by elements of $C_{G}(H)^{f}, f \in F$, we conclude that $G$ has exponent $e$.

\section{Cyclically graded Lie rings with 'selective nilpotency' condition}

In this section we develop a Lie ring theory which is used in Section 5 below for studying groups $G$ and Lie rings $L$ with a metacyclic Frobenius group of automorphisms $F H$. This theory is stated in terms Lie rings with finite cyclic grading, which will arise from the 'eigenspaces' for $F$. By Kreknin's theorem ([13]) the condition

$$
C_{L}(F)=0
$$

implies the solvability of $L$ of derived length bounded in terms of $|F|$, but our aim is to obtain nilpotency of class bounded in terms of $|H|$ and the nilpotency class of $C_{L}(H)$. (Here $C_{L}(F)$ and $C_{L}(H)$ denote the fixed-point subrings for $F$ and $H$.) The nilpotency of $C_{L}(H)$ of class $c$ implies certain restrictions on the commutation of the grading components, which we nickname 'selective nilpotency'. For example, in [9] it was shown that if $c=1$, that is, $C_{L}(H)$ is abelian, and $|F|$ is a prime, then each component commutes with all but at most $(c,|H|)$-boundedly many components, which in turn implies a strong bound for the nilpotency class of $L$. In this section we work with another, rather technical 'selective nilpotency' condition. As we shall see in Section 5, this condition actually arises quite naturally in dealing with a Lie ring admitting a metacyclic Frobenius group of automorphisms; similar conditions were exploited earlier in the paper [19] on double Frobenius groups. In this section we virtually achieve a good $(c,|H|)$-bounded de- 
rived length and Engel-type conditions, which prepare ground for the next section, where nilpotency of $(c,|H|)$-bounded class is finally obtained by using additional considerations.

\section{Numerical preliminaries}

The next two lemmas are elementary facts on polynomials.

Lemma 4.1. Let $K$ be a field of characteristic 0 containing a primitive qth root of unity $\omega$. Suppose that $m=\omega^{i_{1}}+\cdots+\omega^{i_{m}}$ for some positive integer $m$ and some $0 \leqslant i_{1}, \ldots, i_{m} \leqslant q-1$. Then $i_{1}=\cdots=i_{m}=0$.

Proof. Without loss of generality it can be assumed that $K=\mathbb{Q}[\omega]$. Since we have $m=\left|\omega^{i_{1}}\right|+\cdots+\left|\omega^{i_{m}}\right|$, the lemma follows from the triangle inequality.

Lemma 4.2. Let $g_{1}(x)=a_{s} x^{s}+\cdots+a_{0}$ and $g_{2}(x)=b_{t} x^{t}+\cdots+b_{0}$ be polynomials with integer coefficients that have no non-zero common complex roots and let $M=\max _{i, j}\left\{\left|a_{i}\right|,\left|b_{j}\right|\right\}$. Suppose that $n_{0}$ is a positive integer such that $g_{1}$ and $g_{2}$ have a non-zero common root in $\mathbb{Z} / n_{0} \mathbb{Z}$. Then

$$
n_{0} \leqslant 2^{2^{(s+t-1)}-1} M^{2^{(s+t-1)}} .
$$

Proof. We use induction on $s+t$. If one of the polynomials has degree 0 , say, $g_{1}(x)=b$, then $n_{0}$ divides $|b|$ and the statement is correct. Therefore we can assume that $s$ and $t$ are both positive and $a_{s}, b_{t} \neq 0$. Let $s \geqslant t$. We set

$$
p(x)=b_{t} g_{1}(x)-g_{2}(x) a_{s} x^{s-t} .
$$

The polynomial $p(x)$ has degree at most $s-1$ and the polynomials $g_{2}(x)$ and $p(x)$ have a non-zero common root in $\mathbb{Z} / n_{0} \mathbb{Z}$ but not in $\mathbb{C}$. We observe that $p(x)$ is of the form

$$
p(x)=c_{s-1} x^{s-1}+\cdots+c_{0},
$$

where

$$
\begin{array}{ll}
c_{i}=b_{t} a_{i}-a_{s} b_{i-s+t} & \text { for } i=s-t, \ldots, s-1, \\
c_{i}=b_{t} a_{i} & \text { for } i=0, \ldots, s-t-1 .
\end{array}
$$

Let $M_{0}=\max _{i, j}\left\{\left|c_{i}\right|,\left|b_{j}\right|\right\}$. By induction, $n_{0} \leqslant 2^{2^{(s+t-2)}-1} M_{0}{ }^{2^{(s+t-2)}}$. Using that $M_{0} \leqslant 2 M^{2}$ we compute

$$
n_{0} \leqslant 2^{2^{(s+t-2)}-1} M_{0} 2^{2^{(s+t-2)}} \leqslant 2^{2^{(s+t-1)}-1} M^{2^{(s+t-1)}},
$$

as required. 
In Section 5 below we shall consider a Frobenius group $F H$ with cyclic kernel $F=\langle f\rangle$ of order $n$ and (necessarily cyclic) complement $H=\langle h\rangle$ of order $q$ acting as $f^{h}=f^{r}$ for $1 \leqslant r \leqslant n-1$. This is where the following conditions come from for numbers $n, q, r$, which we fix for the rest of this section:

$n, q, r$ are positive integers such that $1 \leqslant r \leqslant n-1$ and the image of $r$

in $\mathbb{Z} / d \mathbb{Z}$ is a primitive $q$ th root of 1 for every divisor $d$ of $n$.

In particular, $q$ divides $d-1$ for every divisor $d$ of $n$. When convenient, we freely identify $r$ with its image in $\mathbb{Z} / n \mathbb{Z}$ and regard $r$ as an element of $\mathbb{Z} / n \mathbb{Z}$ such that $r^{q}=1$.

Definition. Let $a_{1}, \ldots, a_{k}$ be not necessarily distinct non-zero elements of $\mathbb{Z} / n \mathbb{Z}$. We say that the sequence $\left(a_{1}, \ldots, a_{k}\right)$ is $r$-dependent if

$$
a_{1}+\cdots+a_{k}=r^{\alpha_{1}} a_{1}+\cdots+r^{\alpha_{k}} a_{k}
$$

for some elements $\alpha_{i} \in\{0,1,2, \ldots, q-1\}$ not all of which are zero. If the sequence $\left(a_{1}, \ldots, a_{k}\right)$ is not $r$-dependent, we call it $r$-independent.

Remark 4.3. A single non-zero element $a \in \mathbb{Z} / n \mathbb{Z}$ is always $r$-independent: if $a=r^{\alpha} a$ for $\alpha \in\{1,2, \ldots, q-1\}$, then $a=0$ by (4.1).

Notation. For a given $r$-independent sequence $\left(a_{1}, \ldots, a_{k}\right)$ let $D\left(a_{1}, \ldots, a_{k}\right)$ denote the set of all $j \in \mathbb{Z} / n \mathbb{Z}$ such that $\left(a_{1}, \ldots, a_{k}, j\right)$ is $r$-dependent.

Lemma 4.4. If $\left(a_{1}, \ldots, a_{k}\right)$ is $r$-independent, then $\left|D\left(a_{1}, \ldots, a_{k}\right)\right| \leqslant q^{k+1}$.

Proof. Suppose that $\left(a_{1}, \ldots, a_{k}, j\right)$ is $r$-dependent. We have

$$
a_{1}+a_{2}+\cdots+a_{k}+j=r^{i_{1}} a_{1}+r^{i_{2}} a_{2}+\cdots+r^{i_{k}} a_{k}+r^{i_{0}} j
$$

for suitable $0 \leqslant i_{s} \leqslant q-1$, where not all of the $i_{s}$ equal to 0 . In fact, $i_{0} \neq 0$, for otherwise the sequence $\left(a_{1}, \ldots, a_{k}\right)$ would not be $r$-independent. Moreover, $1-r^{i_{0}}$ is invertible because by our assumption (4.1) the image of $r$ in $\mathbb{Z} / d \mathbb{Z}$ is a primitive $q$ th root of 1 for every divisor $d$ of $n$. We see that

$$
j=\left(r^{i_{1}} a_{1}+\cdots+r^{i_{k}} a_{k}-a_{1}-a_{2}-\cdots-a_{k}\right) /\left(1-r^{i_{0}}\right),
$$

so there are at most $q^{k+1}$ possibilities for $j$, as required.

We will need a sufficient condition for a sequence $\left(a_{1}, \ldots, a_{k}\right)$ to contain an $r$-independent subsequence. 
Lemma 4.5. Suppose that for some $m$ a sequence $\left(a_{1}, \ldots, a_{k}\right)$ of non-zero elements of $\mathbb{Z} / n \mathbb{Z}$ contains at least $q^{m}+m$ different values. Then one can choose an $r$-independent subsequence $\left(a_{1}, a_{i_{2}}, \ldots, a_{i_{m}}\right)$ of $m$ elements that contains $a_{1}$.

Proof. If $m=1$, the lemma is obvious, as $a_{1}$ is $r$-independent by Remark 4.3. So we assume that $m \geqslant 2$ and use induction on $m$. By induction we can choose an $r$-independent subsequence of length $m-1$ starting from $a_{1}$. Without loss of generality we can assume that $\left(a_{1}, a_{2}, \ldots, a_{m-1}\right)$ is an $r$-independent subsequence. By Lemma 4.4 there are at most $q^{m}$ distinct elements in $D\left(a_{1}, a_{2}, \ldots, a_{m-1}\right)$. Since there are at least $q^{m}+m$ different values in the sequence $\left(a_{1}, \ldots, a_{k}\right)$, the sequence $a_{m}, a_{m+1} \ldots, a_{k}$ contains at least $q^{m}+1$ different values. So we can always choose an element $b \notin D\left(a_{1}, a_{2}, \ldots, a_{m-1}\right)$ among $a_{m}, a_{m+1} \ldots, a_{k}$ such that the sequence $\left(a_{1}, a_{2}, \ldots, a_{m-1}, b\right)$ is $r$-independent.

\section{'Selective nilpotency' condition on graded Lie algebras}

To recall the definitions of graded Lie algebras, homogeneous elements and commutators, see the beginning of Section 3 . Here we work with a $(\mathbb{Z} / n \mathbb{Z})$-graded Lie ring $L$ such that $L_{0}=0$. Formally the symbol $\mathbb{Z} / n \mathbb{Z}$ here means the additive group $\mathbb{Z} / n \mathbb{Z}$. However, it will be convenient to use the same symbol to denote also the ring $\mathbb{Z} / n \mathbb{Z}$. To avoid overloaded notation, we adopt the following convention.

Index Convention. Henceforth a small Latin letter with an index $i \in \mathbb{Z} / n \mathbb{Z}$ will denote a homogeneous element in the grading component $L_{i}$, with the index only indicating which component this element belongs to: $x_{i} \in L_{i}$. We will not be using numbering indices for elements of the $L_{i}$, so that different elements can be denoted by the same symbol when it only matters which component the elements belong to. For example, $x_{i}$ and $x_{i}$ can be different elements of $L_{i}$, so that $\left[x_{i}, x_{i}\right]$ can be a non-zero element of $L_{2 i}$.

Note that under Index Convention a homogeneous commutator belongs to the component $L_{s}$ with index $s$ equal to the sum of indices of all the elements involved in this commutator.

Definition. Let $n, q, r$ be integers defined by (4.1). We say that a $(\mathbb{Z} / n \mathbb{Z})$-graded Lie ring $L$ satisfies the selective c-nilpotency condition if, under Index Convention,

$$
\left[x_{d_{1}}, x_{d_{2}}, \ldots, x_{d_{c+1}}\right]=0 \quad \text { whenever }\left(d_{1}, \ldots, d_{c+1}\right) \text { is } r \text {-independent. }
$$

Notation. We use the usual notation $(k, l)$ for the greatest common divisor of integers $k, l$. 
Given $b \in \mathbb{Z} / n \mathbb{Z}$, we denote by $o(b)$ the order of $b$ (in the additive group). Thus, $o(b)$ is the least positive integer such that

$$
\underbrace{b+b+\cdots+b}_{o(b)}=0 .
$$

In the following lemmas the condition $L_{0}=0$ ensures that all indices of nontrivial elements are non-zero, so all sequences of such indices are either $r$-dependent or $r$-independent.

Lemma 4.6. Suppose that a $(\mathbb{Z} / n \mathbb{Z})$-graded Lie ring $L$ with $L_{0}=0$ satisfies the selective c-nilpotency condition (4.2), and let $b$ be an element of $\mathbb{Z} / n \mathbb{Z}$ such that

$$
o(b)>2^{2^{2 q-3}-1} c^{2^{2 q-3}} .
$$

Then there are at most $q^{c+1}$ elements $a \in \mathbb{Z} / n \mathbb{Z}$ such that

$$
[L_{a}, \underbrace{L_{b}, \ldots, L_{b}}_{c}] \neq 0 .
$$

Proof. Suppose that

$$
[L_{a}, \underbrace{L_{b}, \ldots, L_{b}}_{c}] \neq 0 .
$$

By (4.2) the sequence

$$
(a, \underbrace{b, \ldots, b}_{c})
$$

must be $r$-dependent and so we have $a+b+\cdots+b=r^{i_{0}} a+r^{i_{1}} b+\cdots+r^{i_{c}} b$ for suitable $0 \leqslant i_{j} \leqslant q-1$, where at least once $i_{j} \neq 0$. First suppose that we have $i_{0}=0$. Then for some $1 \leqslant m \leqslant c$ and some $1 \leqslant j_{1}, \ldots, j_{m} \leqslant q-1$ we have $\left(r^{j_{1}}+\cdots+r^{j_{m}}-m\right) b=0$, where the $j_{s}$ are not necessarily different. Put $m_{0}=r^{j_{1}}+\cdots+r^{j_{m}}-m$ and $n_{0}=\left(n, m_{0}\right)$. Thus, $o(b)$ divides $n_{0}$ and $r^{j_{1}}+\cdots+r^{j_{m}}-m=0$ in $\mathbb{Z} / n_{0} \mathbb{Z}$. We collect terms re-writing $r^{j_{1}}+\cdots+r^{j_{m}}-m$ as $b_{k_{1}} r^{k_{1}}+\cdots+b_{k_{l}} r^{k_{l}}-m$, where $b_{k_{i}}>0, \sum_{i=1}^{l} b_{k_{i}}=m$, and $q>k_{1}>k_{2}>$ $\cdots>k_{l}>0$ are all different. By Lemma 4.1 the polynomials $X^{q-1}+\cdots+X+1$ and $b_{k_{1}} X^{k_{1}}+\cdots+b_{k_{l}} X^{k_{l}}-m$ have no common complex roots. On the other hand, these polynomials have a common non-zero root in $\mathbb{Z} / n_{0} \mathbb{Z}$ : namely, the image of $r$. Therefore, by Lemma 4.2,

$$
n_{0} \leqslant 2^{2^{2 q-3}-1} m^{2^{2 q-3}} \leqslant 2^{2^{2 q-3}-1} c^{2^{2 q-3}}
$$

This yields a contradiction since $n_{0} \geqslant o(b)>2^{2^{2 q-3}-1} c^{2^{2 q-3}}$. 
Hence, $i_{0} \neq 0$. In this case $1-r^{i_{0}}$ is invertible by assumption (4.1). Therefore,

$$
a=\left(r^{i_{1}} b+\cdots+r^{i_{c}} b-c b\right) /\left(1-r^{i_{0}}\right),
$$

so there are at most $q^{c+1}$ possibilities for $a$. The lemma follows.

Lemma 4.7. Suppose that a $(\mathbb{Z} / n \mathbb{Z})$-graded Lie ring $L$ with $L_{0}=0$ satisfies the selective c-nilpotency condition (4.2). There is a $(c, q)$-bounded number $w$ such that

$$
[L, \underbrace{L_{b}, \ldots, L_{b}}_{w}]=0
$$

whenever $b$ is an element of $\mathbb{Z} / n \mathbb{Z}$ such that $o(b)>\max \left\{2^{2^{2 q-3}-1} c^{2^{2 q-3}}, q^{c+1}\right\}$.

Proof. Denote by $N(b)$ the set of all $a \in \mathbb{Z} / n \mathbb{Z}$ such that

$$
[L_{a}, \underbrace{L_{b}, \ldots, L_{b}}_{c}] \neq 0 .
$$

By Lemma 4.6, $N=|N(b)| \leqslant q^{c+1}$. If for some $t \geqslant 1$ we have

$$
[L_{a}, \underbrace{L_{b}, \ldots, L_{b}}_{c+t}] \neq 0,
$$

then all elements $a, a+b, a+2 b, a+t b$ belong to $N(b)$. It follows that either

$$
[L_{a}, \underbrace{L_{b}, \ldots, L_{b}}_{c+N}]=0
$$

or $s b=0$ for some $s \leqslant N$. But the latter case with $o(b) \leqslant N$ is impossible by the hypothesis $o(b)>q^{c+1} \geqslant N$. Hence,

$$
[L, \underbrace{L_{b}, \ldots, L_{b}}_{c+q^{c+1}}]=0 .
$$

Now recall that for a given $r$-independent sequence $\left(a_{1}, \ldots, a_{k}\right)$ we denote by $D\left(a_{1}, \ldots, a_{k}\right)$ the set of all $j \in \mathbb{Z} / n \mathbb{Z}$ such that $\left(a_{1}, \ldots, a_{k}, j\right)$ is $r$-dependent.

Let $\left(d_{1}, \ldots, d_{c}\right)$ be an arbitrary $r$-independent sequence, which we consider as fixed in the next few lemmas.

Lemma 4.8. Suppose that a $(\mathbb{Z} / n \mathbb{Z})$-graded Lie ring $L$ with $L_{0}=0$ satisfies the selective c-nilpotency condition (4.2), and let $U=\left[u_{d_{1}}, \ldots, u_{d_{c}}\right]$ be a homogeneous commutator with the $r$-independent sequence of indices $\left(d_{1}, \ldots, d_{c}\right)$ (under 
Index Convention). Then every commutator of the form

$$
\left[U, x_{i_{1}}, \ldots, x_{i_{t}}\right]
$$

can be written as a linear combination of commutators of the form

$$
\left[U, m_{j_{1}}, \ldots, m_{j_{s}}\right]
$$

where $j_{k} \in D\left(d_{1}, \ldots, d_{c}\right)$ and $s \leqslant t$. The case $s=t$ is possible only if we have $i_{k} \in D\left(d_{1}, \ldots, d_{c}\right)$ for all $k=1, \ldots, t$.

Proof. The assertion is obviously true for $t=0$. Let $t=1$. If $i_{1} \in D\left(d_{1}, \ldots, d_{c}\right)$, then $\left[U, x_{i_{1}}\right]$ is of the required form. If $i_{1} \notin D\left(d_{1}, \ldots, d_{c}\right)$, then $\left[U, x_{i_{1}}\right]=0$ by (4.2) and there is nothing to prove.

Let us assume that $t>1$ and use induction on $t$. If all the indices $i_{j}$ belong to $D\left(d_{1}, \ldots, d_{c}\right)$, then the commutator $\left[U, x_{i_{1}}, \ldots, x_{i_{t}}\right]$ is of the required form with $s=t$. Suppose that in (4.3) there is an element $x_{i_{k}}$ with the index $i_{k}$ that does not belong to $D\left(d_{1}, \ldots, d_{c}\right)$. We choose such an element with $k$ as small as possible and use $k$ as a second induction parameter.

If $k=1$, then the commutator (4.3) is zero and we are done. Thus, suppose that $k \geqslant 2$ and write

$$
\begin{aligned}
{\left[U, \ldots, x_{i_{k-1}}, x_{i_{k}}, \ldots, x_{i_{t}}\right]=[U, \ldots,} & \left.x_{i_{k}}, x_{i_{k-1}}, \ldots, x_{i_{t}}\right] \\
& +\left[U, \ldots,\left[x_{i_{k-1}}, x_{i_{k}}\right], \ldots, x_{i_{t}}\right] .
\end{aligned}
$$

By induction hypothesis, the commutator $\left[U, \ldots,\left[x_{i_{k-1}}, x_{i_{k}}\right], \ldots, x_{i_{t}}\right]$ is a linear combination of commutators of the form (4.4) because it is shorter than (4.3), while the commutator $\left[U, \ldots, x_{i_{k}}, x_{i_{k-1}}, \ldots, x_{i_{t}}\right]$ is a linear combination of commutators of the form (4.4) because the index that does not belong to $D\left(d_{1}, \ldots, d_{c}\right)$ here occurs closer to $U$ than in (4.3).

Corollary 4.9. Let $L$ and $U$ be as in Lemma 4.8. Then the ideal of $L$ generated by $U$ is spanned by commutators of the form (4.4).

In the next lemma we obtain a rather detailed information about the ideal generated by $U$. Basically it says that id $\langle U\rangle$ is generated by commutators of the form (4.4) in which right after $U$ there are boundedly many elements with indices in $D\left(d_{1}, \ldots, d_{c}\right)$ followed only by elements with indices of small additive order in $\mathbb{Z} / n \mathbb{Z}$.

Notation. From now on we fix the $(c, q)$-bounded number

$$
N(c, q)=\max \left\{2^{2^{2 q-3}-1} c^{2^{2 q-3}}, q^{c+1}\right\},
$$

which appears in Lemma 4.7. 
For our fixed $r$-independent sequence $\left(d_{1}, \ldots, d_{c}\right)$, we denote by $A$ the set of all elements $b \in D\left(d_{1}, \ldots, d_{c}\right)$ such that $o(b)>N(c, q)$ and by $B$ the set of all elements $b \in D\left(d_{1}, \ldots, d_{c}\right)$ such that $o(b) \leqslant N(c, q)$. Let $D=\left|D\left(d_{1}, \ldots, d_{c}\right)\right|$ and let $w$ be the number given by Lemma 4.7.

Lemma 4.10. Let $L$ and $U$ be as in Lemma 4.8. The ideal of $L$ generated by $U$ is spanned by commutators of the form

$$
\left[U, m_{i_{1}}, \ldots, m_{i_{u}}, m_{i_{u+1}}, \ldots, m_{i_{v}}\right],
$$

where $u \leqslant(w-1) D$, with $i_{k} \in D\left(d_{1}, \ldots, d_{c}\right)$ for $k \leqslant u$, and $i_{k} \in B$ for $k>u$.

Proof. Let $R$ be the span of all commutators of the form (4.5). It is sufficient to show that every commutator $W=\left[U, m_{j_{1}}, \ldots, m_{j_{s}}\right]$ of the form (4.4) belongs to $R$. If $s \leqslant(w-1) D$, it is clear that $W \in R$, so we assume that $s>(w-1) D$ and use induction on $s$. Write

$$
W=\left[U, m_{j_{1}}, \ldots, m_{j_{t}}, m_{j_{t-1}}, \ldots, m_{j_{s}}\right]+\left[U, m_{j_{1}}, \ldots,\left[m_{j_{t-1}}, m_{j_{t}}\right], \ldots, m_{j_{s}}\right] .
$$

If $j_{t-1}+j_{t} \in D\left(d_{1}, \ldots, d_{c}\right)$, then the second summand is of the form (4.4). Since it is shorter than $W$, it follows that it belongs to $R$ by the induction hypothesis. If $j_{t-1}+j_{t} \notin D\left(d_{1}, \ldots, d_{c}\right)$, then by Lemma 4.8 the second summand is a linear combination of commutators of the form (4.4) each of which is shorter than $W$.

Thus, in either case the second summand in (4.6) belongs to $R$. It follows that the commutator $W$ does not change modulo $R$ under any permutation of the $m_{j_{k}}$. If among the $m_{j_{k}}$ there are at least $w$ elements with the same index $j_{k} \in A$, we move these elements next to each other. Then it follows from Lemma 4.7 that $W=0$. Suppose now that all the indices $j_{k} \in A$ occur less than $w$ times. We place all these elements right after the $U$. This initial segment has length at most $D(w-1)+c$, so the resulting commutator takes the required form (4.5).

Corollary 4.11. Suppose that a $(\mathbb{Z} / n \mathbb{Z})$-graded Lie ring $L$ with $L_{0}=0$ satisfies the selective c-nilpotency condition (4.2), and let $\left(d_{1}, \ldots, d_{c}\right)$ be an $r$-independent sequence. Then the ideal ${ }_{\text {id }}\left\langle\left[L_{d_{1}}, \ldots, L_{d_{c}}\right]\right\rangle$ has $(c, q)$-boundedly many non-trivial components of the induced grading.

Proof. Let $U=\left[u_{d_{1}}, \ldots, u_{d_{c}}\right]$ be an arbitrary homogeneous commutator with the given indices (under Index Convention). Since in (4.5) we have $i_{k} \in D\left(d_{1}, \ldots, d_{c}\right)$ for all $k=1, \ldots, u$, the sum of all indices of the initial segment $\left[U, m_{i_{1}}, \ldots, m_{i_{u}}\right]$ can take at most $D^{u}$ values. Denote by $Y$ the order of $\langle B\rangle$, the subgroup of $\mathbb{Z} / n \mathbb{Z}$ generated by $B$. Clearly, the sum of the remaining indices in (4.5) belongs to $\langle B\rangle$. 
It follows that the sum of all indices in (4.5) can take at most $D^{u} Y$ values. As the order of every element in $B$ is $(c, q)$-bounded and there are only $(c, q)$-boundedly many elements in $B$, it follows that $Y$ is $(c, q)$-bounded. By Lemmas 4.4, 4.7, and 4.10 the number $u$ is also $(c, q)$-bounded. So id $\langle U\rangle$ has $(c, q)$-boundedly many non-trivial components.

It follows from the proofs of Lemmas 4.8 and 4.10 that the set of indices of all possible non-trivial components in id $\langle U\rangle$ is completely determined by the tuple $\left(d_{1}, \ldots, d_{c}\right)$ and does not depend on the choice of $U=\left[u_{d_{1}}, \ldots, u_{d_{c}}\right]$. Since the ideal id $_{\text {id }}\left\langle\left[L_{d_{1}}, \ldots, L_{d_{c}}\right]\right\rangle$ is the sum of ideals id $\left\langle\left[u_{d_{1}}, \ldots, u_{d_{c}}\right]\right\rangle$ over all possible $u_{d_{1}}, \ldots, u_{d_{c}}$, the result follows.

Lemma 4.12. Suppose that a homogeneous ideal $T$ of a Lie ring $L$ has only e nontrivial components. Then $L$ has at most $e^{2}$ components that do not centralize $T$.

Proof. Let $T_{i_{1}}, \ldots, T_{i_{e}}$ be the non-trivial homogeneous components of $T$ and let $S=\left\{i_{1}, \ldots, i_{e}\right\}$. Suppose that $L_{i}$ does not centralize $T$. Then for some $j \in S$ we have $i+j \in S$. So there are at most $|S| \times|S|$ possibilities for $i$, as required.

Proposition 4.13. Suppose that a $(\mathbb{Z} / n \mathbb{Z})$-graded Lie ring $L$ with $L_{0}=0$ satisfies the selective c-nilpotency condition (4.2). Then $L$ is solvable of $(c, q)$-bounded derived length $f(c, q)$.

Note that $L$ is solvable of $n$-bounded derived length by Kreknin's theorem, but we need a bound for the derived length in terms of $c$ and $q$.

Proof. We use induction on $c$. If $c=0$, then $L=0$ and there is nothing to prove. Indeed, $L_{0}=0$ by hypothesis, and for $d \neq 0$ we have $L_{d}=0$ by (4.2), since any element $d \neq 0$ is $r$-independent by Remark 4.3.

Now let $c \geqslant 1$. Let $I$ be the ideal of the given Lie ring $L$ generated by all commutators $\left[L_{i_{1}}, \ldots, L_{i_{c}}\right]$, where $\left(i_{1}, \ldots, i_{c}\right)$ ranges through all $r$-independent sequences of length $c$. The induced $(\mathbb{Z} / n \mathbb{Z})$-grading of $L / I$ has trivial zero-component and $L / I$ satisfies the selective $(c-1)$-nilpotency condition. By the induction hypothesis $L / I$ is solvable of bounded derived length, say, $f_{0}$, that is, $L^{\left(f_{0}\right)} \leqslant I$.

Consider an arbitrary $r$-independent sequence $\left(i_{1}, \ldots, i_{c}\right)$ and the ideal

$$
T={ }_{\text {id }}\left\langle\left[L_{i_{1}}, \ldots, L_{i_{c}}\right]\right\rangle .
$$

We know from Corollary 4.11 that there are only $(c, q)$-boundedly many, say, $e$, non-trivial grading components in $T$. By Lemma 4.12 there are at most $e^{2}$ components that do not centralize $T$. Since $C_{L}(T)$ is also a homogeneous ideal, it follows that the quotient $L / C_{L}(T)$ has at most $e^{2}$ non-trivial components. Since the induced $(\mathbb{Z} / n \mathbb{Z})$-grading of $L / C_{L}(T)$ also has trivial zero component, by Shalev's 
generalization [23] of Kreknin's theorem we conclude that $L / C_{L}(T)$ is solvable of $e$-bounded derived length, say, $f_{1}$. Therefore $L^{\left(f_{1}\right)}$, the corresponding term of the derived series, centralizes $T$. Since $f_{1}$ does not depend on the choice of the $r$-independent tuple $\left(i_{1}, \ldots, i_{c}\right)$ and $I$ is the sum of all such ideals $T$, it follows that $\left[L^{\left(f_{1}\right)}, I\right]=0$. Recall that $L^{\left(f_{0}\right)} \leqslant I$. Hence, $\left[L^{\left(f_{1}\right)}, L^{\left(f_{0}\right)}\right]=0$. Thus $L$ is solvable of $(c, q)$-bounded derived length at $\operatorname{most} \max \left\{f_{0}, f_{1}\right\}+1$.

\section{Combinatorial corollary}

We now state a combinatorial corollary of Lemma 4.7 and Proposition 4.13 that we shall need in the next section for dealing with non-semisimple automorphisms, when eigenspaces do not form a direct sum.

We use the following notation:

$$
\delta_{1}=\left[x_{1}, x_{2}\right], \quad \delta_{k+1}=\left[\delta_{k}\left(x_{1}, \ldots, x_{2^{k}}\right), \delta_{k}\left(x_{2^{k}-1}, \ldots, x_{2^{k+1}}\right)\right] .
$$

Corollary 4.14. Let $n, q, r$ be positive integers such that $1 \leqslant r \leqslant n-1$ and the image of $r$ in $\mathbb{Z} / d \mathbb{Z}$ is a primitive qth root of 1 for every divisor $d$ of $n$.

(a) For the function $f(c, q)$ given by Proposition 4.13 above, the following holds. If we arbitrarily and formally assign lower indices $i_{1}, i_{2}, \ldots \in \mathbb{Z}$ to elements $y_{i_{1}}, y_{i_{2}}, \ldots$ of an arbitrary Lie ring, then the commutator

$$
\delta_{f(c, q)}\left(y_{i_{1}}, y_{i_{2}}, \ldots y_{i_{2} f(q, c)}\right)
$$

can be represented as a linear combination of commutators in the same elements $y_{i_{1}}, y_{i_{2}}, \ldots, y_{i_{2} f(q, c)}$ each of which contains either a subcommutator with zero modulo $n$ sum of indices or a subcommutator of the form

$$
\left[g_{u_{1}}, g_{u_{2}}, \ldots, g_{u_{c+1}}\right]
$$

with an $r$-independent sequence $\left(u_{1}, \ldots, u_{c+1}\right)$ of indices, where elements $g_{j}$ are commutators in $y_{i_{1}}, y_{i_{2}}, \ldots, y_{i_{2} f(c, q)}$ such that the sum of indices of all the elements involved in $g_{j}$ is congruent to $j$ modulo $n$.

(b) For the function $w=w(c, q)$ given by Lemma 4.7, any commutator

$$
[y_{a}, \underbrace{x_{b}, y_{b}, \ldots, z_{b}}_{w}]
$$

with $w$ elements with the same index $b$ over the brace, can be represented as a linear combination of the same form as in (a) whenever the image of $b$ in $\mathbb{Z} / n \mathbb{Z}$ is such that $o(b)>N(c, q)=\max \left\{2^{2^{2 q-3}-1} c^{2^{2 q-3}}, q^{c+1}\right\}$. 
Proof. (a) Let $M$ be a free Lie ring freely generated by $y_{i_{1}}, y_{i_{2}}, \ldots, y_{i_{2} f(c, q)}$. We define for each $i=0,1, \ldots, n-1$ the additive subgroup $M_{i}$ of $M$ generated (in the additive group) by all commutators in the generators $y_{i_{j}}$ with the sum of indices congruent to $i$ modulo $n$. Then, obviously, $M=M_{0} \oplus M_{1} \oplus \cdots \oplus M_{n-1}$ and $\left[M_{i}, M_{j}\right] \subseteq M_{i+j}(\operatorname{modn})$, so that this is a $(\mathbb{Z} / n \mathbb{Z})$-grading. By Proposition 4.13 we obtain

$$
\begin{aligned}
\delta_{f(c, q)}\left(y_{i_{1}}, y_{i_{2}}, \ldots, y_{i_{2} f(c, q)}\right) \\
\in \in_{\text {id }}\left\langle M_{0}\right\rangle+\sum_{\substack{\left(u_{1}, \ldots, u_{c+1}\right) \\
\text { is } r \text {-independent }}} \text { id }\left\{\left[M_{u_{1}}, M_{u_{2}}, \ldots, M_{u_{c+1}}\right]\right\rangle .
\end{aligned}
$$

By the definition of $M_{i}$ this inclusion is equivalent to the required equality in the conclusion of Corollary 4.14 (a). Since the elements $y_{i_{1}}, y_{i_{2}}, \ldots y_{i_{2} f(c, q)}$ freely generate the Lie ring $M$, the same equality holds in any Lie ring.

(b) The proof of the second statement is obtained by the same arguments with the only difference that Lemma 4.7 is applied instead of Proposition 4.13.

\section{Bounding the nilpotency class}

In this section we obtain bounds for the nilpotency class of groups and Lie rings admitting a metacyclic Frobenius group of automorphisms with fixed-point-free kernel. Earlier such results were obtained by the second and third authors [19] in the case of the kernel of prime order. Examples at the end of the section show that such nilpotency results are no longer true for non-metacyclic Frobenius groups of automorphisms. The results for groups are consequences of the corresponding results for Lie rings and algebras, by various Lie ring methods.

\section{Lie algebras}

We begin with the theorem for Lie algebras, which is devoid of technical details required for arbitrary Lie rings, so that the ideas of proof are more clear. Recall that $C_{L}(A)$ denotes the fixed-point subalgebra for a group of automorphisms $A$ of a Lie algebra $L$, and that nilpotency class is also known as nilpotency index.

Theorem 5.1. Let FH be a Frobenius group with cyclic kernel $F$ of order $n$ and complement $H$ of order $q$. Suppose that FH acts by automorphisms on a Lie algebra $L$ in such a way that $C_{L}(F)=0$ and $C_{L}(H)$ is nilpotent of class $c$. Then $L$ is nilpotent of $(c, q)$-bounded class.

Note that the assumption of the kernel $F$ being cyclic is essential: see the corresponding examples at the end of the section. 
Proof. Our first aim is to reduce the problem to the study of $\mathbb{Z} / n \mathbb{Z}$-graded Lie algebras satisfying the selective $c$-nilpotency condition (4.2). Then the solubility of $L$ of $(c, q)$-bounded derived length will immediately follow from Proposition 4.13. Further arguments are then applied to obtain nilpotency.

Let $\omega$ be a primitive $n$th root of 1 . We extend the ground field by $\omega$ and denote the resulting Lie algebra by $\widetilde{L}$. The group $F H$ acts in a natural way on $\widetilde{L}$ and this action inherits the conditions that $C_{\widetilde{L}}(F)=0$ and $C_{\widetilde{L}}(H)$ is nilpotent of class $c$. Thus, we can assume that $L=\widetilde{L}$ and the ground field contains $\omega$, a primitive $n$th root of 1 .

We shall need a simple remark that we can assume that $n=|F|$ is not divisible by the characteristic of the ground field. Indeed, if the characteristic $p$ divides $n$, then the Hall $p^{\prime}$-subgroup $\langle\chi\rangle$ of $F$ acts fixed-point-freely on $L$-otherwise the Sylow $p$-subgroup $\langle\psi\rangle$ of $F$ would have non-trivial fixed points on the $\psi$-invariant subspace $C_{L}(\chi)$, and these would be non-trivial fixed points for $F$. Thus, $L$ admits the Frobenius group of automorphisms $\langle\chi\rangle H$ with $C_{L}(\chi)=0$. Replacing $F$ by $\langle\chi\rangle$ we can assume that $p$ does not divide $n$.

Let $\varphi$ be a generator of $F$. For each $i=0, \ldots, n-1$ we denote by

$$
L_{i}=\left\{x \in L \mid x^{\varphi}=\omega^{i} x\right\}
$$

the eigenspace for the eigenvalue $\omega^{i}$. Then

$$
\left[L_{i}, L_{j}\right] \subseteq L_{i+j(\operatorname{modn})} \quad \text { and } \quad L=\bigoplus_{i=0}^{n-1} L_{i},
$$

so this is a $(\mathbb{Z} / n \mathbb{Z})$-grading. We also have $L_{0}=C_{L}(F)=0$.

Since $F$ is cyclic of order $n, H$ is also cyclic. Let $H=\langle h\rangle$ and let $\varphi^{h^{-1}}=\varphi^{r}$ for some $1 \leqslant r \leqslant n-1$. Then $r$ is a primitive $q$ th root of 1 in $\mathbb{Z} / n \mathbb{Z}$, and, moreover, the image of $r$ in $\mathbb{Z} / d \mathbb{Z}$ is a primitive $q$ th root of 1 for every divisor $d$ of $n$, since $h$ acts fixed-point-freely on every subgroup of $F$. Thus, $n, q, r$ satisfy condition (4.1).

The group $H$ permutes the components $L_{i}$ so that $L_{i}{ }^{h}=L_{r i}$ for all $i \in \mathbb{Z} / n \mathbb{Z}$. Indeed, if $x_{i} \in L_{i}$, then

$$
\left(x_{i}^{h}\right)^{\varphi}=x_{i}^{h \varphi h^{-1} h}=\left(x_{i}^{\varphi^{r}}\right)^{h}=\omega^{i r} x_{i}^{h} .
$$

Given $u_{k} \in L_{k}$, we temporarily denote $u_{k}^{h^{i}}$ by $u_{r^{i} k}$ (under Index Convention). The sum over any $H$-orbit belongs to $C_{L}(H)$ and therefore

$$
u_{k}+u_{r k}+\cdots+u_{r^{q-1} k} \in C_{L}(H) .
$$


Let $x_{a_{1}}, \ldots, x_{a_{c+1}}$ be homogeneous elements in $L_{a_{1}}, \ldots, L_{a_{c+1}}$, respectively. Consider the elements

$$
\begin{aligned}
X_{1} & =x_{a_{1}}+x_{r a_{1}}+\cdots+x_{r^{q-1} a_{1}}, \\
& \vdots \\
X_{c+1} & =x_{a_{c+1}}+x_{r a_{c+1}}+\cdots+x_{r^{q-1} a_{c+1}} .
\end{aligned}
$$

Since all of them lie in $C_{L}(H)$, which is nilpotent of class $c$, it follows that

$$
\left[X_{1}, \ldots, X_{c+1}\right]=0 .
$$

After expanding the expressions for the $X_{i}$, we obtain on the left a linear combination of commutators in the elements $x_{r^{j}} a_{i}$, which in particular involves the term $\left[x_{a_{1}}, \ldots, x_{a_{c+1}}\right]$. Suppose that the commutator $\left[x_{a_{1}}, \ldots, x_{a_{c+1}}\right]$ is non-zero. Then there must be other terms in the expanded expression that belong to the same component $L_{a_{1}+\cdots+a_{c+1}}$. In other words, then

$$
a_{1}+\cdots+a_{c+1}=r^{\alpha_{1}} a_{1}+\cdots+r^{\alpha_{c+1}} a_{c+1}
$$

for some $\alpha_{i} \in\{0,1,2, \ldots, q-1\}$ not all of which are zeros, so that $\left(a_{1}, \ldots, a_{c+1}\right)$ is an $r$-dependent sequence. This means that $L$ satisfies the selective $c$-nilpotency condition (4.2). By Proposition 4.13 we obtain that $L$ is solvable of $(c, q)$-bounded derived length.

We now use induction on the derived length to prove that $L$ is nilpotent of $(c, q)$-bounded class. If $L$ is abelian, there is nothing to prove. Assume that $L$ is metabelian - this is, in fact, the main part of the proof. When $L$ is metabelian, we have $[x, y, z]=[x, z, y]$ for every $x \in[L, L]$ and $y, z \in L$. The key step is a stronger version of Lemma 4.7 for the metabelian case, without the arithmetical condition on the additive orders.

Lemma 5.2. Let $L$ be metabelian. There is a $(c, q)$-bounded number $m$ such that

$$
[L, \underbrace{L_{b}, \ldots, L_{b}}_{m}]=0 \text { for every } b \in \mathbb{Z} / n \mathbb{Z} .
$$

Proof. For each $a \in \mathbb{Z} / n \mathbb{Z}$ we denote $[L, L] \cap L_{a}$ by $[L, L]_{a}$. Clearly, it suffices to show that

$$
[[L, L]_{a}, \underbrace{L_{b}, \ldots, L_{b}}_{m-1}]=0 \quad \text { for every } a, b \in \mathbb{Z} / n \mathbb{Z}
$$

we can of course assume that $a, b \neq 0$. First suppose that $(n, b)=1$. If $n$ is large enough, $n>N(c, q)$, then the order $o(b)=n$ is also large enough and the result 
follows by Lemma 4.7 with $m-1=w$, where $w$ is given by Lemma 4.7. If, however, $n \leqslant N(c, q)$, then we find a positive integer $k<n$ such that $a+k b=0$ in $\mathbb{Z} / n \mathbb{Z}$. (As usual, we freely switch from considering positive integers to their images in $\mathbb{Z} / n \mathbb{Z}$ without changing notation.) Then

$$
[L_{a}, \underbrace{L_{b}, \ldots, L_{b}}_{k}] \subseteq L_{0}=0,
$$

so that we can put $m-1=N(c, q)$.

Now suppose that $(n, b) \neq 1$, and let $s$ be a prime dividing both $n$ and $b$. If $s$ also divides $a$, then the result follows by induction on $|F|$ applied to the Lie algebra $C_{L}\left(\varphi^{n / s}\right)=\sum_{i} L_{s i}$ containing both $L_{a}$ and $L_{b}$ : this Lie algebra is $F H$-invariant and $\varphi$ acts on it as an automorphism of order $n / s$ without non-trivial fixed points. The basis of this induction is the case of $n=|F|$ being a prime, where necessarily $(n, b)=1$, which has already been dealt with above. (Alternatively, we can refer to the main result of [19], where Theorem 5.1 was proved for $F$ of prime order.) Note that the function $m=m(c, q)$ remains the same in the induction step, so no dependence on $|F|$ arises.

Thus, it remains to consider the case where the prime $s$ divides both $b$ and $n$ and does not divide $a$. Using the same notation $x_{r^{i} k}=x_{k}^{h^{i}}$ (under Index Convention), we have

$$
\begin{aligned}
& {\left[u_{a}+u_{r a}+\cdots+u_{r^{q-1} a},\right.} \\
& \quad \underbrace{\left(v_{b}+v_{r b}+\cdots+v_{r^{q-1} b}\right), \ldots,\left(w_{b}+w_{r b}+\cdots+w_{r^{q-1} b}\right)}_{c}]=0
\end{aligned}
$$

for any $c$ elements $v_{b}, \ldots, w_{b} \in L_{b}$, because here sums are elements of $C_{L}(H)$.

By (4.1) any two indices $r^{i} a, r^{j} a$ here are different modulo $s$, while all the indices above the brace are divisible by $s$, which divides $n$. Hence we also have

$$
[u_{a}, \underbrace{\left(v_{b}+v_{r b}+\cdots+v_{r^{q-1} b}\right), \ldots,\left(w_{b}+w_{r b}+\cdots+w_{r^{q-1} b}\right)}_{c}]=0 .
$$

Let $Z$ denote the span of all the sums $x_{b}+x_{r b}+\cdots+x_{r^{q-1} b}$ over $x_{b} \in L_{b}$ (in fact, $Z$ is the fixed-point subspace of $H$ on $\left.\bigoplus_{i=0}^{q-1} L_{r^{i} b}\right)$. Then (5.1) means that

$$
[L_{a}, \underbrace{Z, \ldots, Z}_{c}]=0 \text {. }
$$

Applying $\varphi^{j}$ we also obtain

$$
[L_{a}, \underbrace{Z^{\varphi^{j}}, \ldots, Z^{\varphi^{j}}}_{c}]=[L_{a}^{\varphi^{j}}, \underbrace{Z^{\varphi^{j}}, \ldots, Z^{\varphi^{j}}}_{c}]=0 .
$$


A Vandermonde-type linear algebra argument shows that $L_{b} \subseteq \sum_{j=0}^{q-1} Z^{\varphi^{j}}$. We prove this fact in a greater generality, which will be needed later.

Lemma 5.3. Let $\langle\varphi\rangle$ be a cyclic group of order $n$, and let $\omega$ be a primitive $n$th root of unity. Suppose that $M$ is a $\mathbb{Z}[\omega]\langle\varphi\rangle$-module such that $M=\sum_{i=1}^{m} M_{k_{i}}$, where $x \varphi=\omega^{k_{i}} x$ for $x \in M_{k_{i}}$ and $0 \leqslant k_{1}<k_{2}<\cdots<k_{m}<n$. If

$$
z=y_{k_{1}}+y_{k_{2}}+\cdots+y_{k_{m}} \text { for } y_{k_{i}} \in M_{k_{i}} \text {, }
$$

then for some $m$-bounded number $l_{0}$ every element $n^{l_{0}} y_{k_{s}}$ is a $\mathbb{Z}[\omega]$-linear combination of the elements $z, z \varphi, \ldots, z \varphi^{m-1}$.

Proof. We have

$$
z \varphi^{j}=\omega^{j k_{1}} y_{k_{1}}+\omega^{j k_{2}} y_{k_{2}}+\cdots+\omega^{j k_{m}} y_{k_{m}} .
$$

Giving values $j=0, \ldots, m-1$, we obtain $m$ linear combinations of the elements $y_{k_{1}}, y_{k_{2}}, \ldots, y_{k_{m}}$. Then for every $s=1, \ldots, m$ a suitable linear combination of these linear combinations produces $D y_{k_{s}}$, where $D$ is the Vandermonde determinant of the $m \times m$ matrix of coefficients of these linear combinations, which is equal to

$$
\prod_{1 \leqslant i<j \leqslant m}\left(\omega^{k_{i}}-\omega^{k_{j}}\right) .
$$

Each factor is a product of an (invertible) power of $\omega$ and an element $1-\omega^{k_{i}-k_{j}}$. By hypothesis the exponent $k_{i}-k_{j}$ is not divisible by $n$, so $\rho=\omega^{k_{i}-k_{j}}$ is a root of the polynomial $1+x+\cdots+x^{n-1}=(x-\rho) g(x)$. By substituting $x=1$ we obtain that $n=(1-\rho) g(1)$, so by multiplying $D y_{k_{s}}$ by an invertible power of $\omega$ and several elements of the type $g(1)$ we obtain $n^{l_{0}} y_{k_{s}}$ for some $m$-bounded number $l_{0}$. As a result, $n^{l_{0}} y_{k_{s}}$ is expressed as a $\mathbb{Z}[\omega]$-linear combination of the elements $z, z \varphi, \ldots, z \varphi^{m-1}$.

In the proof of Lemma 5.2 we can apply Lemma 5.3 with

$$
M=L_{b}+L_{r b}+\cdots+L_{r^{q-1} b} \text { and } m=q
$$

to $w=v_{b}+v_{r b}+\cdots+v_{r^{q-1} b} \in Z$ for any $v_{b} \in L_{b}$, because here all the indices $r^{i} b$ can be regarded as pairwise distinct residues modulo $n$ by condition (4.1) (that $r$ is a primitive $q$ th root of unity modulo any divisor of $n$ ). Using the fact that $n$ is invertible in our ground field, we obtain that $L_{b} \subseteq \sum_{j=0}^{q-1} Z^{\varphi^{j}}$.

We now claim that

$$
[[L, L]_{a}, \underbrace{L_{b}, \ldots, L_{b}}_{q(c-1)+1}]=0 .
$$


Indeed, after replacing $L_{b}$ with $\sum_{j=0}^{q-1} Z^{\varphi^{j}}$ and expanding the sums, in each commutator of the resulting linear combination we can freely permute the entries $Z^{\varphi^{j}}$, since $L$ is metabelian. Since there are sufficiently many of them, we can place at least $c$ of the same $Z^{\varphi^{j_{0}}}$ for some $j_{0}$ right after $[L, L]_{a}$ at the beginning, which gives 0 by (5.2).

We now return to the case of metabelian $L$ in the proof of Theorem 5.1. Let $m=m(c, q)$ be as in Lemma 5.2 and put $g=(m-1)\left(q^{c+1}+c\right)+2$. For any sequence of $g$ non-zero elements $\left(a_{1}, \ldots, a_{g}\right)$ in $\mathbb{Z} / n \mathbb{Z}$ consider the commutator $\left[[L, L]_{a_{1}}, L_{a_{2}}, \ldots, L_{a_{g}}\right]$. If the sequence $\left(a_{1}, \ldots, a_{g}\right)$ contains an $r$-independent subsequence of length $c+1$ that starts with $a_{1}$, by permuting the $L_{a_{i}}$ we can assume that $a_{1}, \ldots, a_{c+1}$ is the $r$-independent subsequence. Then

$$
\left[[L, L]_{a_{1}}, L_{a_{2}}, \ldots, L_{a_{g}}\right]=0
$$

by (4.2). If the sequence $\left(a_{1}, \ldots, a_{g}\right)$ does not contain an $r$-independent subsequence of length $c+1$ starting with the element $a_{1}$, then by Lemma 4.5 the sequence $\left(a_{1}, \ldots, a_{g}\right)$ contains at most $q^{c+1}+c$ different values. The number $g$ was chosen big enough to guarantee that either the value of $a_{1}$ occurs in $\left(a_{1}, \ldots, a_{g}\right)$ at least $m+1$ times or, else, another value, different from $a_{1}$, occurs at least $m$ times. Using that $[x, y, z]=[x, z, y]$ for $x \in[L, L]$ we can assume that

$$
a_{2}=\cdots=a_{m+1},
$$

in which case it follows from Lemma 5.2 that

$$
\left[[L, L]_{a_{1}}, L_{a_{2}}, \ldots, L_{a_{g}}\right]=0 .
$$

Thus, we conclude that $L$ is nilpotent of class at most $g$.

Now suppose that the derived length of $L$ is at least 3. By induction hypothesis, $[L, L]$ is nilpotent of bounded class. According to the previous paragraph, the quotient $L /[[L, L],[L, L]]$ is nilpotent of bounded class, as well. Together, this gives nilpotency of $L$ of bounded class by the Lie ring analogue (3.1) of P. Hall's theorem.

\section{Lie groups and torsion-free locally nilpotent groups}

We now derive the group-theoretic consequences of Theorem 5.1; but the theorem on finite groups will have to wait until we prove a similar result for Lie rings. By the well-known connection between Lie groups and their Lie algebras, the following theorem is an immediate consequence of Theorem 5.1. Recall that $C_{G}(A)$ denotes the fixed-point subgroup for a group of automorphisms $A$ of a group $G$, and that nilpotency class is also known as nilpotency index. 
Theorem 5.4. Suppose that a connected Lie group $G$ (complex or real) admits a finite Frobenius group of automorphisms $F H$ with cyclic kernel $F$ of order $n$ and complement $H$ of order $q$ such that $C_{G}(F)=1$ and $C_{G}(H)$ is nilpotent of class $c$. Then $G$ is nilpotent of $(c, q)$-bounded class.

Proof. Every automorphism $\alpha$ of $G$ induces the automorphism $d_{e} \alpha$ of the tangent Lie algebra $g$ of $G$ which is the differential of $\alpha$ at identity. The fixed-point subalgebra $C_{\mathrm{g}}\left(d_{e} \alpha\right)$ is the tangent Lie algebra of the fixed-point subgroup $C_{G}(\alpha)$ (see, for example, [21, Theorem 3.7]). Therefore the group of automorphisms $\overline{F H}$ of $\mathrm{g}$ induced by $F H$ has the properties that $C_{\mathfrak{g}}(\bar{F})=0$ and $C_{\mathfrak{g}}(\bar{H})$ is nilpotent of class $c$. (This can also be shown by using the Exp and Log functors, which locally commute with automorphisms of $G$.) By Theorem 5.1, the Lie algebra $g$ is nilpotent of $(c, q)$-bounded class. Since our Lie group $G$ is connected, this implies the same result for $G$.

Another corollary of Theorem 5.1 follows by a similar Lie ring method - the Mal'cev correspondence, which is also based on the Exp and Log functors and the Baker-Campbell-Hausdorff formula.

Theorem 5.5. Suppose that a locally nilpotent torsion-free group $G$ admits a finite Frobenius group of automorphisms $F H$ with cyclic kernel $F$ of order $n$ and complement $H$ of order $q$ such that $C_{G}(F)=1$ and $C_{G}(H)$ is nilpotent of class $c$. Then $G$ is nilpotent of $(c, q)$-bounded class.

Proof. It is clearly sufficient to prove the theorem for finitely generated $F H$-invariant subgroups of $G$, so we can assume that $G$ is finitely generated and therefore nilpotent. Let $\widehat{G}$ be the Mal'cev completion of $G$ obtained by adjoining all roots of non-trivial elements of $G$ (see [20] or, for example, [8, Section 10.1]). Forming the completion preserves the nilpotency class of a nilpotent subgroup. Every automorphism $\alpha$ of $G$ can be canonically extended to an automorphism of $\widehat{G}$, which we denote by the same letter. The fixed-point subgroup $C_{\widehat{G}}(\alpha)$ is the completion of $C_{G}(\alpha)$. Applying this to $F H$, we see that as a group of automorphisms of $\widehat{G}$, it inherits the properties that $C_{\widehat{G}}(F)=1$ and $C_{\widehat{G}}(H)$ is nilpotent of class $c$.

Under the Mal'cev correspondence, the radicable locally nilpotent torsion-free group $\widehat{G}$ can be viewed as a locally nilpotent Lie algebra $L$ (with the same underlying set) over the field of rational numbers $\mathbb{Q}$, with the Lie ring operations given by the inversions of the Baker-Campbell-Hausdorff formula. The automorphisms of $\widehat{G}$ are automorphisms of $L$ acting on the same set in the same way, and we denote them by the same letters. Thus, $C_{L}(F)=0$ and $C_{L}(H)$ is nilpotent of class $c$. By Theorem 5.1 the Lie algebra $L$ is nilpotent of $(c, q)$-bounded class. Hence the same is true for $\widehat{G}$ and therefore also for $G$. 


\section{Lie rings}

We now prove a similar theorem for arbitrary Lie rings. The additional conditions on the additive group of the Lie ring will be automatically satisfied when the theorem is later used in the proof of the main result on the nilpotency class of a finite group with a metacyclic Frobenius group of automorphisms. We also include the solvability result, which does not require those additional conditions.

Theorem 5.6. Let FH be a Frobenius group with cyclic kernel $F$ of order $n$ and complement $H$ of order $q$. Suppose that $F H$ acts by automorphisms on a Lie ring $L$ in such a way that $C_{L}(F)=0$ and $C_{L}(H)$ is nilpotent of class $c$. Then

(a) the Lie ring $L$ is solvable of $(c, q)$-bounded derived length,

(b) for some functions $u=u(c, q)$ and $v=v(c, q)$ depending only on $c$ and $q$, the Lie subring $n^{u} L$ is nilpotent of class $v$, that is,

$$
\gamma_{v+1}\left(n^{u} L\right)=n^{u(v+1)} \gamma_{v+1}(L)=0,
$$

(c) moreover, $L$ is nilpotent of $(c, q)$-bounded class in either of the following cases:

(i) L is a Lie algebra over a field,

(ii) the additive group of $L$ is periodic (includes the case of $L$ finite),

(iii) $n$ is invertible in the ground ring of $L$,

(iv) $n L=L$,

(v) $n$ is a prime-power.

We included for completeness the case of algebras over a field, which is Theorem 5.1. It is not clear at the moment if the additional conditions (i)-(v) are really necessary. However, many important cases are covered, including those needed in our group-theoretic applications. The solvability result is included precisely because it does not require these conditions: of course, $L$ is solvable of $n$-bounded derived length by Kreknin's theorem, but we obtain a bound for the derived length in terms of $|H|$ and the nilpotency class of $C_{L}(H)$.

Proof. The proof is basically along the same lines as the proof of Theorem 5.1 for algebras; the complications arise because we no longer have a direct sum of eigenspaces forming a $(\mathbb{Z} / n \mathbb{Z})$-grading.

We extend the ground ring by a primitive $n$th root of unity $\omega$ setting

$$
\widetilde{L}=L \otimes_{\mathbb{Z}} \mathbb{Z}[\omega]
$$


The group $F H$ acts in the natural way on $\widetilde{L}$ and the action inherits the conditions that $C_{\widetilde{L}}(F)=0$ and $C_{\widetilde{L}}(H)$ is nilpotent of class $c$. Since the conditions (i)-(v) would also hold for $\widetilde{L}$, and the conclusion of the theorem for $L$ would follow from the same conclusion for $\widetilde{L}$, we can assume that $L=\widetilde{L}$ and the ground ring contains $\omega$, a primitive $n$th root of 1 .

Let $F=\langle\varphi\rangle$. For each $i=0, \ldots, n-1$ we define the 'eigenspace' for $\omega^{i}$ as $L_{i}=\left\{x \in L \mid x^{\varphi}=\omega^{i} x\right\}$. Then

$$
\left[L_{i}, L_{j}\right] \subseteq L_{i+j}(\bmod n) \quad \text { and } \quad n L \subseteq \sum_{i=0}^{n-1} L_{i} .
$$

This is 'almost a $(\mathbb{Z} / n \mathbb{Z})$-grading' - albeit of $n L$ rather than of $L$, and although the sum is not necessarily direct, any linear dependence of elements from different $L_{i}$ is annihilated by $n$ :

$$
\text { if } \quad l_{1}+l_{2}+\cdots+l_{n-1}=0, \text { then } n l_{1}=n l_{2}=\cdots=n l_{n-1}=0
$$

(see, for example, [7, Lemma 4.1.1]). We also have $L_{0}=C_{L}(F)=0$.

We shall mostly work with the $F H$-invariant subring $K=\sum_{i=0}^{n-1} L_{i}$.

As in the proof of Theorem 5.1, let $H=\langle h\rangle$ and

$$
\varphi^{h^{-1}}=\varphi^{r} \quad \text { for some } 1 \leqslant r \leqslant n-1,
$$

so that $n, q, r$ satisfy (4.1) and $L_{i}{ }^{h}=L_{r i}$ for all $i \in \mathbb{Z} / n \mathbb{Z}$.

Using the same notation

$$
u_{r^{i} k}=u_{k}^{h^{i}} \quad \text { and } \quad X_{j}=x_{a_{j}}+x_{r a_{j}}+\cdots+x_{r^{q-1} a_{j}} \in C_{L}(H),
$$

we have

$$
\left[X_{1}, \ldots, X_{c+1}\right]=0 .
$$

Expand all the $X_{i}$ and consider the resulting linear combination of commutators in the elements $x_{r^{j} a_{i}}$. Suppose that $\left[x_{a_{1}}, \ldots, x_{a_{c+1}}\right] \neq 0$. In the 'semisimple' case, we find that the sum $K=\sum_{i=0}^{n-1} L_{i}$ is direct and there would have to be other terms in the same component $L_{a_{1}+\cdots+a_{c+1}}$, which implies that

$$
a_{1}+\cdots+a_{c+1}=r^{\alpha_{1}} a_{1}+\cdots+r^{\alpha_{c+1}} a_{c+1}
$$

for some $\alpha_{i} \in\{0,1,2, \ldots, q-1\}$ not all of which are zeros, so that $\left(a_{1}, \ldots, a_{c+1}\right)$ is an $r$-dependent sequence. This would mean that $K=\sum_{i=0}^{n-1} L_{i}$ satisfies the selective $c$-nilpotency condition (4.2).

In the general case, where $K$ may not be the direct sum of the $L_{i}$, it can happen that there are no other terms in the expanded expression with the same sum of 
indices $a_{1}+\cdots+a_{c+1}$. But then the 'almost linear independence' (5.3) implies that $n\left[x_{a_{1}}, \ldots, x_{a_{c+1}}\right]=0$. Thus, in any case,

$$
n\left[x_{d_{1}}, x_{d_{2}}, \ldots, x_{d_{c+1}}\right]=0 \quad \text { whenever }\left(d_{1}, \ldots, d_{c+1}\right) \text { is } r \text {-independent. (5.4) }
$$

We are now ready to prove part (a) on solvability. Let $y_{i_{j}} \in L_{i_{j}}$ be any elements of the 'eigenspaces' (under Index Convention). By Corollary 4.14 (a) the commutator $\delta_{f(c, q)}\left(y_{i_{1}}, y_{i_{2}}, \ldots, y_{i_{2} f(c, q)}\right)$ can be represented as a linear combination of commutators each of which contains either a subcommutator with zero modulo $n$ sum of indices or a subcommutator $\left[g_{d_{1}}, g_{d_{2}}, \ldots, g_{d_{c+1}}\right]$ with $r$-independent sequence $\left(d_{1}, \ldots, d_{c+1}\right)$ of indices. By hypothesis we have $L_{0}=0$, and $n\left[g_{d_{1}}, g_{d_{2}}, \ldots, g_{d_{c+1}}\right]=0$ by property (5.4). Hence,

$$
n \delta_{f(c, q)}\left(y_{i_{1}}, y_{i_{2}}, \ldots, y_{i_{2} f(q, c)}\right)=0 .
$$

Thus, $n K^{(f(c, q))}=0$ and therefore

$$
n(n L)^{f(c, q))}=n^{2^{f(c, q)}+1} L^{(f(c, q))}=0 .
$$

In particular, the additive group of $T=L^{(f(c, q))}$ is a periodic abelian group. We decompose it into the direct sum of Sylow subgroups

$$
T=T_{p_{1}} \oplus T_{p_{2}} \oplus \cdots \oplus T_{p_{r}},
$$

where $p_{1}, p_{2}, \ldots, p_{r}$ are the prime divisors of $n$. The $T_{p_{k}}$ are $F H$-invariant ideals and $\left[T_{p_{i}}, T_{p_{j}}\right]=0$ for $i \neq j$.

Let $p \in\left\{p_{1}, p_{2}, \ldots, p_{r}\right\}$ and $n=p^{k} s$, where $(p, s)=1$. Let $\langle\psi\rangle$ be the Sylow $p$-subgroup of $F=\langle\varphi\rangle$ and let $\langle\varphi\rangle=\langle\psi\rangle \times\langle\chi\rangle$, where $s=|\chi|$. The fixed-point subring $C=C_{T_{p}}(\chi)$ is a $\psi$-invariant abelian $p$-group and $C_{C}(\psi) \subseteq C_{L}(\varphi)=0$. The automorphism $\psi$ of order $p^{t}$ acting on an abelian $p$-group necessarily has non-trivial fixed points. Hence we get $C_{T_{p}}(\chi)=0$. Thus the subring $T_{p}$ admits the Frobenius group of automorphisms $\langle\chi\rangle H$ with cyclic kernel $\langle\chi\rangle$ and complement $H$ of order $q$ such that $C_{T_{p}}(\chi)=0$ and $C_{T_{p}}(H)$ is nilpotent of class at most $c$. By the above argument,

$$
0=s\left(s T_{p}\right)^{(f(c, q))}=s^{2^{f(c, q)}+1} T_{p}^{(f(c, q))},
$$

whence $T_{p}^{(f(c, q))}=0$ for each prime $p$. Hence

$$
T^{(f(c, q))}=\left(L^{(f(c, q))}\right)^{(f(c, q))}=0
$$

and $L$ is solvable of derived length at most $2 f(c, q)$. The proof of part (a) is complete.

In the proof of the nilpotency statements, we need an analogue of Lemma 5.2. 
Lemma 5.7. There are $(c, q)$-bounded numbers $l$ and $m$ such that for every element $b \in \mathbb{Z} / n \mathbb{Z}$

$$
n^{l}[K, \underbrace{L_{b}, \ldots, L_{b}}_{m}] \subseteq[[K, K],[K, K]] .
$$

Proof. We denote $[K, K] \cap L_{a}$ by $[K, K]_{a}$. Clearly, it suffices to show that

$$
n^{l}[[K, K]_{a}, \underbrace{L_{b}, \ldots, L_{b}}_{m-1}] \subseteq[[K, K],[K, K]]
$$

for every $a, b \in \mathbb{Z} / n \mathbb{Z}$; we can of course assume that $a, b \neq 0$. First suppose that $(n, b)=1$. If $n$ is large enough, $n>N(c, q)$, then the order $o(b)=n$ is also large enough. Then by Corollary 4.14 (b), for the corresponding function $w=w(c, q)$, any commutator

$$
[y_{a}, \underbrace{x_{b}, y_{b}, \ldots, z_{b}}_{w}]
$$

can be represented as a linear combination of commutators each of which has either a subcommutator with zero modulo $n$ sum of indices or a subcommutator $\left[g_{u_{1}}, g_{u_{2}}, \ldots, g_{u_{c+1}}\right]$ with $r$-independent sequence of indices $\left(u_{1}, \ldots, u_{c+1}\right)$. Since $L_{0}=0$ and $n\left[g_{u_{1}}, g_{u_{2}}, \ldots, g_{u_{c+1}}\right]=0$ by property (5.4), we obtain that

$$
n[[K, K]_{a}, \underbrace{L_{b}, \ldots, L_{b}}_{m-1}]=0,
$$

so that we can put $m-1=w$. If $n \leqslant N(c, q)$, then there is a positive integer $k<n$ such that $a+k b=0$ in $\mathbb{Z} / n \mathbb{Z}$. Then

$$
[L_{a}, \underbrace{L_{b}, \ldots, L_{b}}_{k}] \subseteq L_{0}=0,
$$

so that we can put $m-1=N(c, q)$.

Now suppose that $(n, b) \neq 1$, and let $s$ be a prime dividing both $n$ and $b$. If $s$ also divides $a$, then the result follows by induction on $|F|$ applied to the Lie ring $C_{L}\left(\varphi^{n / s}\right)=\sum_{i} L_{s i}$ containing both $L_{a}$ and $L_{b}$ : this Lie subring is $F H$-invariant and $\varphi$ acts on it as an automorphism of order $n / s$ without non-trivial fixed points. The basis of this induction is the case of $n=|F|$ being a prime, where necessarily $(n, b)=1$, which has already been dealt with above. The functions $l=l(c, q)$ and $m=m(c, q)$ remain the same in the induction step, so no dependence on $F$ arises.

It remains to consider the case where the prime $s$ divides both $b$ and $n$ and does not divide $a$. Using the same notation $x_{r^{i} k}=x_{k}^{h^{i}}$ (under Index Convention), we 
have

$$
\begin{aligned}
& {\left[u_{a}+u_{r a}+\cdots+u_{r^{q-1} a},\right.} \\
& \quad \underbrace{\left(v_{b}+v_{r b}+\cdots+v_{r^{q-1} b}\right), \ldots,\left(w_{b}+w_{r b}+\cdots+w_{r^{q-1} b}\right)}_{c}]=0
\end{aligned}
$$

for any $c$ elements $v_{b}, \ldots, w_{b} \in L_{b}$, because the sums are elements of $C_{L}(H)$.

By (4.1) any two indices $r^{i} a, r^{j} a$ here are different modulo $s$, while all the indices above the brace are divisible by $s$, which divides $n$. Hence by the 'almost linear independence' (5.3) we also have

$$
n[u_{a}, \underbrace{\left(v_{b}+v_{r b}+\cdots+v_{r^{q-1} b}\right), \ldots,\left(w_{b}+w_{r b}+\cdots+w_{r^{q-1} b}\right)}_{c}]=0 .
$$

Now, let $Z$ denote the additive subgroup of $\sum_{i=0}^{q-1} L_{r^{i} b}$ generated by all the sums $x_{b}+x_{r b}+\cdots+x_{r^{q-1} b}$ over $x_{b} \in L_{b}$ (in general $Z$ may not contain all the fixed points of $H$ on $\sum_{i=0}^{q-1} L_{r^{i} b}$ ). Then (5.5) means that

$$
n[L_{a}, \underbrace{Z, \ldots, Z}_{c}]=0 .
$$

Applying $\varphi^{j}$ we also obtain

$$
n[L_{a}, \underbrace{Z^{\varphi^{j}}, \ldots, Z^{\varphi^{j}}}_{c}]=0 .
$$

We now apply Lemma 5.3 with $M=L_{b}+L_{r b}+\cdots+L_{r^{q-1} b}$ and $m=q$ to $w=v_{b}+v_{r b}+\cdots+v_{r^{q-1} b} \in Z$ for any $v_{b} \in L_{b}$. As a result we obtain that $n^{l_{0}} L_{b} \subseteq \sum_{j=0}^{q-1} Z^{\varphi^{j}}$ for some $(c, q)$-bounded number $l_{0}$.

We now claim that

$$
\begin{aligned}
n^{l_{0}(q(c-1)+1)+1}[[K, K]_{a}, \underbrace{L_{b}, \ldots, L_{b}}_{q(c-1)+1}] & =n[[K, K]_{a}, \underbrace{n^{l_{0}} L_{b}, \ldots, n^{l_{0}} L_{b}}_{q(c-1)+1}] \\
& \subseteq[[K, K],[K, K]] .
\end{aligned}
$$

Indeed, after replacing $n^{l_{0}} L_{b}$ with $\sum_{j=0}^{q-1} Z^{\varphi^{j}}$ and expanding the sums, in each commutator of the resulting linear combination we can freely permute modulo $[[K, K],[K, K]]$ the entries $Z^{\varphi^{j}}$. Since there are sufficiently many of them, we can place at least $c$ of the same $Z^{\varphi^{j_{0}}}$ for some $j_{0}$ right after $[L, L]_{a}$ at the beginning, which gives 0 by (5.6). 
We now prove part (b) of the theorem. Since $L$ is solvable of $(c, q)$-bounded derived length by (a), we can use induction on the derived length of $L$. If $L$ is abelian, there is nothing to prove. Assume that $L$ is metabelian, which is the main part of the proof. Consider $K=\sum L_{i}$. Let $l=l(c, q)$ and $m=m(c, q)$ be as in Lemma 5.7; put $g=(m-1)\left(q^{c+1}+c\right)+2$. For any sequence of $g$ non-zero elements $\left(a_{1}, \ldots, a_{g}\right)$ in $\mathbb{Z} / n \mathbb{Z}$ consider the commutator $\left[[K, K]_{a_{1}}, L_{a_{2}}, \ldots, L_{a_{g}}\right]$. If the sequence $\left(a_{1}, \ldots, a_{g}\right)$ contains an $r$-independent subsequence of length $c+1$ that starts with $a_{1}$, by permuting the $L_{a_{i}}$ we can assume that $a_{1}, \ldots, a_{c+1}$ is the $r$-independent subsequence. Then $n\left[[K, K]_{a_{1}}, L_{a_{2}}, \ldots, L_{a_{g}}\right]=0$ by (5.4). If the sequence $\left(a_{1}, \ldots, a_{g}\right)$ does not contain an $r$-independent subsequence of length $c+1$ starting with $a_{1}$, then by Lemma 4.5 the sequence $\left(a_{1}, \ldots, a_{g}\right)$ contains at most $q^{c+1}+c$ different values. The number $g$ was chosen big enough to guarantee that either the value of $a_{1}$ occurs in $\left(a_{1}, \ldots, a_{g}\right)$ at least $m+1$ times or, else, another value, different from $a_{1}$, occurs at least $m$ times. Using the fact that $[x, y, z]=[x, z, y]$ for $x \in[K, K]$, we can assume that $a_{2}=\cdots=a_{m+1}$, in which case it follows from Lemma 5.7 that $n^{l}\left[[K, K]_{a_{1}}, L_{a_{2}}, \ldots, L_{a_{g}}\right]=0$. Thus, in any case $n^{l} \gamma_{g+1}(K)=0$. Clearly, we can also choose a $(c, q)$-bounded number $l_{1}$ such that $\gamma_{g+1}\left(n^{l_{1}} K\right)=0$. As $n L \subseteq K$, we get $\gamma_{g+1}\left(n^{l_{1}+1} L\right)=0$, as required.

Now suppose that the derived length of $L$ is at least 3. By induction hypothesis, $\gamma_{g^{\prime}+1}\left(n^{l_{2}}[L, L]\right)=0$ for some $(c, q)$-bounded numbers $g^{\prime}$ and $l_{2}$. Since $g \geqslant 4$, we can choose a $(c, q)$-bounded number $y$ such that $y(g+1-4) \geqslant(g+1)\left(l_{1}+1\right)$ $\Leftrightarrow y(g+1) \geqslant\left(l_{1}+1\right)(g+1)+4 y$. We set $x=\max \left\{y, l_{2} / 2\right\}$. Then for $M=n^{x} L$ we have both

$$
\begin{aligned}
\gamma_{g+1}(M) & =\gamma_{g+1}\left(n^{x} L\right)=n^{x(g+1)} \gamma_{g+1}(L) \\
& \subseteq n^{\left(l_{1}+1\right)(g+1)+4 x} \gamma_{g+1}(L) \subseteq n^{4 x}[[L, L],[L, L]] \\
& =[[M, M],[M, M]],
\end{aligned}
$$

by the metabelian case above, and

$$
\gamma_{g^{\prime}+1}([M, M])=\gamma_{g^{\prime}+1}\left(n^{2 x}[L, L]\right) \subseteq \gamma_{g^{\prime}+1}\left(n^{l_{2}}[L, L]\right)=0 .
$$

Together, these give the nilpotency of $M=n^{x} L$ of $(c, q)$-bounded class by the Lie ring analogue (3.1) of P. Hall's theorem.

We now proceed with nilpotency of $L$ itself in cases (i)-(v) of part (3).

Case (i). The case of a Lie algebra was already settled in Theorem 5.1.

Cases (iii) and (iv). In these two cases we have $L=n L=n^{i} L$ for any $i$. By part (b),

$$
\gamma_{v+1}(L)=\gamma_{v+1}\left(n^{u} L\right)=0 .
$$


Case (v). Let $n=p^{k}$ for a prime $p$. Then

$$
\gamma_{v+1}\left(\left(p^{k}\right)^{u} L\right)=p^{k u(v+1)} \gamma_{v+1}(L)=0
$$

by part (b), so that the additive group $\gamma_{v+1}(L)$ is a $p$-group. Since in this case $F$ is a cyclic $p$-group acting fixed-point-freely, we must have $\gamma_{v+1}(L)=0$.

Case (ii). Since here the additive group of $L$ is periodic, it decomposes into the direct sum of Sylow subgroups $L=\bigoplus_{p} T_{p}$, which are $F H$-invariant ideals satisfying $\left[T_{q}, T_{p}\right]=0$ for different prime numbers $p, q$. It is sufficient to prove that every ideal $T_{p}$ is nilpotent of $(c, q)$-bounded class. So we can assume that the additive group of $L$ is a $p$-group. If $p$ does not divide $n$, then $n L=L$ and the assertion follows from (iv). If $p$ divides $n$, then the Hall $p^{\prime}$-subgroup $\langle\chi\rangle$ of $F$ acts fixedpoint-freely on $L$, so that $L$ admits the Frobenius group of automorphisms $\langle\chi\rangle H$ with $C_{L}(\chi)=0$. Since $|\chi| L=L$, it remains to apply (iv) to $\langle\chi\rangle H$.

\section{Finite groups}

As a consequence we obtain our main result on the bound for the nilpotency class of a finite group with a metacyclic Frobenius group of automorphisms.

Theorem 5.8. Suppose that a finite group $G$ admits a Frobenius group of automorphisms $F H$ with cyclic kernel $F$ of order $n$ and complement $H$ of order $q$ such that $C_{G}(F)=1$ and $C_{G}(H)$ is nilpotent of class $c$. Then $G$ is nilpotent of $(c, q)$-bounded class.

The assumption of the kernel $F$ being cyclic is essential: see the examples at the end of the section.

Proof. The group $G$ is nilpotent by Theorem 2.7 (c). We have to bound the nilpotency class of $G$ in terms of $c$ and $q$. Consider the associated Lie ring of $G$

$$
L(G)=\bigoplus_{i=1}^{m} \gamma_{i} / \gamma_{i+1},
$$

where $m$ is the nilpotency class of $G$ and the $\gamma_{i}$ are terms of the lower central series of $G$. The nilpotency class of $G$ coincides with that of $L(G)$. The action of the group $F H$ on $G$ naturally induces an action of $F H$ on $L(G)$. By the definition of the associated Lie ring,

$$
C_{L(G)}(H)=\bigoplus_{i} C_{\gamma_{i} / \gamma_{i+1}}(H),
$$


which by Theorem 2.3 is equal to

$$
\bigoplus_{i} C_{\gamma_{i}}(H) \gamma_{i+1} / \gamma_{i+1}
$$

The Lie ring products in $L(G)$ are defined for elements of the $\gamma_{i} / \gamma_{i+1}$ in terms of the images of the group commutators and then extended by linearity. If $c$ is the nilpotency class of $C_{G}(H)$, then any group commutator of weight $c+1$ in elements of $C_{G}(H)$ is trivial; hence any Lie ring commutator of weight $c+1$ in elements of $C_{\gamma_{i}}(H) \gamma_{i+1} / \gamma_{i+1}$ is also trivial. Since these elements generate $C_{L(G)}(H)$, this subring is nilpotent of class at most $c$. Because $F$ acts fixed-point-freely on every quotient $\gamma_{i} / \gamma_{i+1}$ by Lemma 2.2, it follows that $C_{L(G)}(F)=0$.

We now deduce from Theorem 5.6 (c) that $L(G)$ is nilpotent of $(c, q)$-bounded class. Since the nilpotency class of our group $G$ is equal to that of $L(G)$, the result follows.

\section{Examples}

Example 5.9. The simple 3 -dimensional Lie algebra $L$ of characteristic $\neq 2$ with basis $e_{1}, e_{2}, e_{3}$ and structure constants $\left[e_{1}, e_{2}\right]=e_{3},\left[e_{2}, e_{3}\right]=e_{1},\left[e_{3}, e_{1}\right]=e_{2}$ admits the Frobenius group of automorphisms $F H$ with $F$ non-cyclic of order 4 and $H$ of order 3 such that $C_{L}(F)=0$ and $C_{L}(H)$ is one-dimensional. Namely, $F=\left\{1, f_{1}, f_{2}, f_{3}\right\}$, where $f_{i}\left(e_{i}\right)=e_{i}$ and $f_{i}\left(e_{j}\right)=-e_{j}$ for $i \neq j$, and $H=\langle h\rangle$ with $h\left(e_{i}\right)=e_{i+1(\bmod 3)}$.

Example 5.10. One can also modify the above example to produce nilpotent Lie rings $L$ of unbounded derived length admitting the same non-metacyclic Frobenius group of automorphisms $F H$ of order 12 such that $C_{L}(F)=0$ and $C_{L}(H)$ is abelian ('one-dimensional'). Namely, let the additive group of $L$ be the direct sum of three copies of $\mathbb{Z} / p^{m} \mathbb{Z}$ for some prime $p \neq 2$ with generators $e_{1}, e_{2}, e_{3}$ and let the structure constants of $L$ be $\left[e_{1}, e_{2}\right]=p e_{3},\left[e_{2}, e_{3}\right]=p e_{1},\left[e_{3}, e_{1}\right]=p e_{2}$. The action of $F H$ is 'the same', that is, $F=\left\{1, f_{1}, f_{2}, f_{3}\right\}$, where $f_{i}\left(e_{i}\right)=e_{i}$ and $f_{i}\left(e_{j}\right)=-e_{j}$ for $i \neq j$, and $H=\langle h\rangle$ with $h\left(e_{i}\right)=e_{i+1(\bmod 3)}$. Then we have $C_{L}(F)=0$ and $C_{L}(H)=\left\langle e_{1}+e_{2}+e_{3}\right\rangle$. It is easy to see that $L$ is nilpotent of class exactly $m$, while the derived length is approximately $\log m$.

Example 5.11. If in the preceding example we choose $p>m$, then the Lazard correspondence based on the 'truncated' Baker-Campbell-Hausdorff formula (cf. [15]; also see, for example, [8, Section 10.2]) transforms $L$ into a finite $p$-group $P$ of the same derived length as $L$, which admits the same group of automorphisms $F H$ with $C_{P}(F)=1$ and $C_{P}(H)$ cyclic. 


\section{Bibliography}

[1] V. V. Belyaev and B. Hartley, Centralizers of finite nilpotent subgroups in locally finite groups, Algebra Logika 35 (1996), 389-410 (in Russian); English translation in Algebra Logic 35 (1996), 217-228.

[2] C. W. Curtis and I. Reiner, Representation Theory of Finite Groups and Associative Algebras, Interscience, New York, London, 1962.

[3] E. C. Dade, Carter subgroups and Fitting heights of finite solvable groups, Illinois J. Math. 13 (1969), 449-514.

[4] J. D. Dixon, M. P. F. du Sautoy, A. Mann and D. Segal, Analytic Pro- $p$ Groups, London Mathematical Society Lecture Note Series 157, Cambridge University Press, Cambridge, 1991.

[5] D. Gorenstein, Finite Groups, Harper and Row, New York, 1968.

[6] P. Hall, Some sufficient conditions for a group to be nilpotent, Illinois J. Math. 2 (1958), 787-801.

[7] E. I. Khukhro, Nilpotent Groups and Their Automorphisms, Walter de Gruyter, Berlin, 1993.

[8] E. I. Khukhro, p-Automorphisms of Finite p-Groups, London Mathematical Society Lecture Note Series 246, Cambridge University Press, Cambridge, 1998.

[9] E. I. Khukhro, Graded Lie rings with many commuting components and an application to 2-Frobenius groups, Bull. Lond. Math. Soc. 40 (2008), 907-912.

[10] E. I. Khukhro, Fixed points of the complements of Frobenius groups of automorphisms, Sibirsk. Mat. Zh., 51 (2010), 694-699 (in Russian); English translation in Sib. Math. J. 51 (2010), 554-558.

[11] E. I. Khukhro and P. V. Shumyatsky, Bounding the exponent of a finite group with automorphisms, J. Algebra 212 (1999), 363-374.

[12] L. G. Kovács, On finite soluble groups, Math. Z. 103 (1968), 37-39.

[13] V. A. Kreknin, The solubility of Lie algebras with regular automorphisms of finite period, Dokl. Akad. Nauk SSSR 150 (1963), 467-469 (in Russian); English translation in Math. USSR Doklady 4 (1963), 683-685.

[14] M. Kuzucuoglu and P. Shumyatsky, On local finiteness of periodic residually finite groups, Proc. Edinb. Math. Soc. (2) 45 (2002), 717-721.

[15] M. Lazard, Sur les groupes nilpotents et les anneaux de Lie, Ann. Sci. Éc. Norm. Supér. 71 (1954), 101-190.

[16] M. Lazard, Groupes analytiques p-adiques, Publ. Math. Inst. Hautes Études Sci. 26 (1965), 389-603.

[17] A. Lubotzky and A. Mann, Powerful p-groups. I: Finite groups, J. Algebra 105 (1987), 484-505. 
[18] N. Y. Makarenko, E. I. Khukhro and P. Shumyatsky, Fixed points of Frobenius groups of automorphisms, to appear in Dokl. Akad. Nauk.

[19] N. Y. Makarenko and P. Shumyatsky, Frobenius groups as groups of automorphisms, Proc. Amer. Math. Soc. 138 (2010), 3425-3436.

[20] A. I. Mal'cev, Nilpotent torsion-free groups, Izv. Akad. Nauk. SSSR Ser. Mat. 13 (1949), 201-212 (in Russian).

[21] A. L. Onishchik and È. B. Vinberg, Lie Groups and Lie Algebras I. Foundations of Lie Theory. Lie Transformation Groups, Springer-Verlag, Berlin, Heidelberg, 1993.

[22] D. F. S. Robinson, A Course in the Theory of Groups, Springer-Verlag, New York, Heidelberg, Berlin, 1982.

[23] A. Shalev, Automorphisms of finite groups of bounded rank, Israel J. Math. 82 (1993), 395-404.

[24] A. G. R. Stewart, On the class of certain nilpotent groups, Proc. R. Soc. Lond. Ser. A Math. Phys. Sci. 292 (1966), 374-379.

[25] Unsolved Problems in Group Theory. The Kourovka Notebook, no. 17, Institute of Mathematics, Novosibirsk, 2010.

Received August 18, 2011.

\section{Author information}

Evgeny Khukhro, Sobolev Institute of Mathematics, Novosibirsk, 630 090, Russia.

E-mail: khukhro@yahoo.co.uk

Natalia Makarenko, Sobolev Institute of Mathematics, Novosibirsk, 630 090, Russia, and Université de Haute Alsace, Mulhouse, 68093, France.

E-mail: natalia_makarenko@yahoo.fr

Pavel Shumyatsky, Department of Mathematics, University of Brasilia,

DF 70910-900, Brazil.

E-mail: pavel@mat.unb.br 